\title{
A unitarity compatible approach to one-loop amplitudes with massive fermions
}

\section{Simon Badger, ${ }^{a}$ Christian Brønnum-Hansen, ${ }^{b}$ Francesco Buciuni ${ }^{a}$ and Donal O'Connell ${ }^{b}$}

\author{
${ }^{a}$ Institute for Particle Physics Phenomenology, Ogden Centre for Fundamental Physics, \\ Department of Physics, University of Durham, \\ Science Laboratories, South Rd, Durham DH1 3LE, U.K. \\ ${ }^{b}$ Higgs Centre for Theoretical Physics, \\ School of Physics and Astronomy, The University of Edinburgh, \\ James Clerk Maxwell Building, Peter Guthrie Tait Road, Edinburgh EH9 3FD, U.K. \\ E-mail: simon.d.badger@durham.ac.uk, bronnum.hansen@ed.ac.uk, \\ francesco.buciuni@durham.ac.uk, donal@staffmail.ed.ac.uk
}

ABSTRACT: We explain how one-loop amplitudes with massive fermions can be computed using only on-shell information. We first use the spinor-helicity formalism in six dimensions to perform generalised unitarity cuts in $d$ dimensions. We then show that divergent wavefunction cuts can be avoided, and the remaining ambiguities in the renormalised amplitudes can be fixed, by matching to universal infrared poles in $4-2 \epsilon$ dimensions and ultraviolet poles in $6-2 \epsilon$ dimensions. In the latter case we construct an effective Lagrangian in six dimensions and reduce the additional constraint to an on-shell tree-level computation.

KeYwords: Perturbative QCD, Scattering Amplitudes, Field Theories in Higher Dimensions, Effective Field Theories

ARXiv EPRINT: 1703.05734 


\section{Contents}

1 Introduction 1

2 Review of one-loop amplitudes and integrands 3

3 Massive fermion spinors 5

3.1 Massive fermions from massless six dimensional spinors 6

$\begin{array}{ll}3.2 & \text { Interactions and state-sum reduction }\end{array}$

$\begin{array}{ll}3.3 \text { A one-loop example calculation } & 10\end{array}$

4 Generalised unitarity cuts in six dimensions $\quad 12$

5 Determining the remaining integral coefficients $\quad 17$

5.1 Fixing $c_{2, m^{2}}$ by matching the poles in $4-2 \epsilon$ dimensions 17

$\begin{array}{lll}5.2 & \text { Counterterms for QCD in six dimensions } & 18\end{array}$

5.3 Fixing $c_{1}$ by matching poles in $6-2 \epsilon$ dimensions 22

6 Conclusions 24

$\begin{array}{ll}\text { A Conventions and spinor construction } & 25\end{array}$

B Tree-level amplitudes in six dimensions $\quad 26$

B.1 Three-point amplitudes 26

$\begin{array}{ll}\text { B.2 } & \text { Four-point amplitudes } \\ \end{array}$

$\begin{array}{ll}\text { C Cut solutions in six dimensions } & 27\end{array}$

$\begin{array}{ll}\text { C.1 Triple cut } & 27\end{array}$

$\begin{array}{lll}\text { C.2 Double cut } & 28\end{array}$

D Feynman rules for the effective Lagrangian 28

\section{Introduction}

Precise predictions for the production of strongly interacting massive particles are in high demand for current experimental analyses at the LHC. The current precision level of predictions is in relatively good shape, with top quark pair production now known differentially at NNLO in QCD $[1,2]$ and a full range of off-shell decays known at NLO in QCD with an additional jet [3]. Modern one-loop techniques are also able to explore high multiplicity final states where the current state-of-the-art is top quark pair production in association 


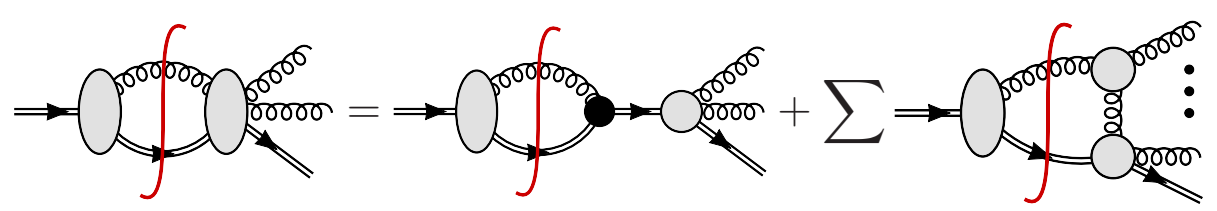

Figure 1. Decomposing the tree amplitude appearing on the left hand side of a wavefunction cut reveals a divergent graph.

with three jets [4]. The GoSam collaboration has also been able to produce NLO predictions for the challenging $t \bar{t} H+j$ final state [5]. A more complete overview of the current status can be found in reference [6].

On the other hand, these processes are often overlooked by more formal studies of amplitudes in gauge theory which can uncover hidden simplicity and structure. While it is well known that on-shell techniques like unitarity [7], spinor integration [8, 9] and BCFW recursion apply equally well to massive amplitudes, explicit computations are relatively few [10-12]. Nevertheless some computations using these approaches have produced compact analytic results useful for phenomenological applications [11, 13]. While elements of these computations use unitarity cuts and on-shell trees, Feynman diagrams techniques were also employed to compute the UV counterterms necessary for mass and wavefunction renormalisation. To the best of our knowledge the only computations not to do this are those with a massive internal loop where a UV matching prescription was used $[7,10]$.

The obstacle is that the traditional approach to renormalisation requires the amputation of wavefunction graphs, and the addition of counterterm diagrams. This procedure breaks gauge invariance during intermediate steps and therefore causes problems for methods based on (generalised) unitarity [14-16], which construct amplitudes from on-shell tree-level building blocks. Naive attempts to amputate wavefunction graphs in generalised unitarity are precluded by the presence of an on-shell propagator, leading to a factor $1 / 0$ : this is depicted explicitly in figure 1, where the on-shell tree amplitude appearing on the right hand side of a two-particle cut is expanded to reveal a divergent propagator inside. Consequently, the favoured method is still to follow an approach based on Feynman diagrams; then the amputation of wavefunction graphs is straightforward.

Two solutions to this problem have been proposed. Ellis, Giele, Kunszt and Melnikov showed that modifying the tree-level input entering the double cuts of the wavefunction graphs allowed a simple implementation of the on-shell renormalisation scheme [17]. All cuts can then be performed but gauge invariance is only restored at the end of the computation. Since the removal of the unwanted graphs is extremely easy to implement within a BerendsGiele construction of the tree-level amplitudes in the cuts this method is quite efficient numerically. A second solution, proposed by Britto and Mirabella [18], is to regulate the divergent tree by introducing a momentum shift. This procedure allows us to preserve gauge invariance but introduces an additional variable into the calculation which will cancel when combined with the mass-renormalisation counterterms. In either case a set of extra twoand single-particle cuts is necessary together with the counterterms to fully determine the amplitude in comparison to the massless case. 
Despite both of these solutions there is still an open question: is it possible to compute amplitudes with masses using only on-shell gauge invariant building blocks and without introducing additional regulators? Both of the approaches mentioned above follow the onshell renormalisation scheme where divergences can be absorbed into additional terms in the Lagrangian. In this paper we will seek an alternative way to absorb the divergences by appealing to an effective six dimensional version of QCD.

This procedure relies on first computing a full set of finite $d$-dimensional unitarity cuts. We show how this can be done efficiently in the six-dimensional spinor-helicity formalism [19] by embedding the additional mass into the higher dimensions and performing cuts in six dimensions. In particular we show how these results can be dimensionally reduced to $d$-dimensional amplitudes keeping the spin dimension of the gluon $d_{s}$ arbitrary. ${ }^{1}$ This generalises the previous approaches used for massless cuts in six-dimensions [20, 21].

Our paper is organised as follows. We begin by reviewing the structure of one-loop amplitudes in dimensional regularisation and the integrand level representations. In section 3 we review the spinor-helicity formalisms in four and six dimensions, and show how Dirac spinors for massive fermions can be represented as massless Weyl spinors in six dimensions. We then discuss a simple example of a pair of massive fermions coupling to an off-shell vertex at one-loop. This example allows us to show how computations in six dimensions can be performed, and how they can be dimensionally reduced to results with an arbitrary spin dimension $d_{s}$. In section 4 we summarise the generalised unitarity method in six dimensions and explain some of the key features needed to apply it to the case of $g g \rightarrow t \bar{t}$ scattering. Section 5 describes the procedure of fixing the remaining ambiguities using the universal epsilon pole structure in $d=4-2 \epsilon$ dimensions and the corresponding epsilon pole structure of the effective theory in $6-2 \epsilon$ dimensions. Following our conclusions, the appendices give further details of the spinor-helicity method, the tree-level amplitudes in six dimensions and multiple cut solutions. We also include an appendix with the Feynman rules for the dimension six operators used in section 5 .

In addition we provide a MATHEMATICA workbook as supplementary material to this article. This workbook contains a basic implementation of all the ingredients used in the paper and runs through numerical examples for two leading colour primitive one-loop amplitudes contributing to $g g \rightarrow t \bar{t}$ scattering. Analytic formulae for these two primitive amplitudes are also provided in a computer readable form including the full dependence on the loop momentum dimension $d$ and spin dimension $d_{s}$.

\section{Review of one-loop amplitudes and integrands}

In this section we review the basics of one-loop integrand parametrisations in $d$ dimensions. While many good reviews of the subject are available, e.g. [22-24], this section also introduces the notation we will use in the rest of the paper. The amplitudes we will consider in this paper are QCD amplitudes with one massive fermion flavour. In this case there are

\footnotetext{
${ }^{1}$ The distinction between the spin dimension, $d_{s}$, and spacetime dimension, $d$, is motivated by different regularisation schemes. We find it to be very convenient to maintain the distinction throughout our calculations. To be clear, we define the spin dimension such that the gluon has $d_{s}-2$ physical polarization states.
} 
only two possible basis integrals which go beyond those appearing in the massless case,

$$
A_{n}^{(1)}=B_{n}^{(1)}+c_{2 ; m^{2}} I_{2, m^{2}}+c_{1} I_{1} .
$$

The amplitude labelled $B_{n}^{(1)}$ is the part that can be constructed from finite $d$-dimensional unitarity cuts. The additional basis integrals depend only on the fermion mass and in dimensional regularisation are,

$$
\begin{aligned}
I_{2, m^{2}} & =\mu_{R}^{2 \epsilon} \int \frac{d^{d} k}{(2 \pi)^{d}} \frac{1}{k^{2}\left((k-p)^{2}-m^{2}\right)} \stackrel{d=\underline{4-2 \epsilon}}{=} i c_{\Gamma}\left(\frac{1}{\epsilon}+\log \left(\frac{\mu_{R}^{2}}{m^{2}}\right)+2\right)+\mathcal{O}(\epsilon), \\
I_{1} & =\mu_{R}^{2 \epsilon} \int \frac{d^{d} k}{(2 \pi)^{d}} \frac{1}{k^{2}-m^{2}} \stackrel{d=\underline{4-2 \epsilon}}{=} i c_{\Gamma} m^{2}\left(\frac{1}{\epsilon}+\log \left(\frac{\mu_{R}^{2}}{m^{2}}\right)+1\right)+\mathcal{O}(\epsilon),
\end{aligned}
$$

where $c_{\Gamma}=\frac{\Gamma(1+\epsilon) \Gamma(1-\epsilon)^{2}}{(4 \pi)^{2-\epsilon} \Gamma(1-2 \epsilon)}$.

The amplitudes $B_{n}^{(1)}$ can be written in the usual integrand basis of irreducible scalar products including extra dimensional terms following the OPP [25]/EGKM [26, 27] constructions,

$$
\begin{aligned}
B_{n}^{(1)}=\mu_{R}^{2 \epsilon} \int \frac{d^{d} k}{(2 \pi)^{d}}\left\{\sum_{1 \leq i_{1}<i_{2}<i_{3}<i_{4}<i_{5} \leq n} \frac{\Delta_{\left\{i_{1}, i_{2}, i_{3}, i_{4}, i_{5}\right\}}}{D_{i_{1}} D_{i_{2}} D_{i_{3}} D_{i_{4}} D_{i_{5}}}+\sum_{1 \leq i_{1}<i_{2}<i_{3}<i_{4} \leq n} \frac{\Delta_{\left\{i_{1}, i_{2}, i_{3}, i_{4}\right\}}}{D_{i_{1}} D_{i_{2}} D_{i_{3}} D_{i_{4}}}\right. \\
\left.+\sum_{1 \leq i_{1}<i_{2}<i_{3} \leq n} \frac{\Delta_{\left\{i_{1}, i_{2}, i_{3}\right\}}}{D_{i_{1}} D_{i_{2}} D_{i_{3}}}+\sum_{\substack{1 \leq i_{1}<i_{2} \leq n \\
i_{2}-i_{1} \bmod n>1}} \frac{\Delta_{\left\{i_{1}, i_{2}\right\}}}{D_{i_{1}} D_{i_{2}}}\right\} .
\end{aligned}
$$

For renormalisable gauge theories we can give a complete parametrisation of the numerators,

$$
\begin{aligned}
\Delta_{\left\{i_{1}, i_{2}, i_{3}, i_{4}, i_{5}\right\}}= & c_{i_{1}, i_{2}, i_{3}, i_{4}, i_{5}}^{(0)} \mu^{2}, \\
\Delta_{\left\{i_{1}, i_{2}, i_{3}, i_{4}\right\}}= & c_{i_{1}, i_{2}, i_{3}, i_{4}}^{(0)}+c_{i_{1}, i_{2}, i_{3}, i_{4}}^{(1)}\left(k \cdot w_{1 ; i_{1}, i_{2}, i_{3}}\right)+c_{i_{1}, i_{2}, i_{3}, i_{4}}^{(2)} \mu^{2} \\
& +c_{i_{1}, i_{2}, i_{3}, i_{4}}^{(3)} \mu^{2}\left(k \cdot w_{1 ; i_{1}, i_{2}, i_{3}}\right)+c_{i_{1}, i_{2}, i_{3}, i_{4}}^{(4)} \mu^{4}, \\
\Delta_{\left\{i_{1}, i_{2}, i_{3}\right\}}= & c_{i_{1}, i_{2}, i_{3}}^{(0)}+c_{i_{1}, i_{2}, i_{3}}^{(1)}\left(k \cdot w_{1 ; i_{1}, i_{2}}\right)+c_{i_{1}, i_{2}, i_{3}}^{(2)}\left(k \cdot w_{2 ; i_{1}, i_{2}}\right) \\
& +c_{i_{1}, i_{2}, i_{3}}^{(3)}\left(k \cdot w_{1 ; i_{1}, i_{2}}\right)\left(k \cdot w_{2 ; i_{1}, i_{2}}\right)+c_{i_{1}, i_{2}, i_{3}}^{(4)}\left(\left(k \cdot w_{1 ; i_{1}, i_{2}}\right)^{2}-\left(k \cdot w_{2 ; i_{1}, i_{2}}\right)^{2}\right) \\
& +c_{i_{1}, i_{2}, i_{3}}^{(5)}\left(k \cdot w_{1 ; i_{1}, i_{2}}\right)^{2}\left(k \cdot w_{2 ; i_{1}, i_{2}}\right)+c_{i_{1}, i_{2}, i_{3}}^{(6)}\left(k \cdot w_{1 ; i_{1}, i_{2}}\right)\left(k \cdot w_{2 ; i_{1}, i_{2}}\right)^{2} \\
& +c_{i_{1}, i_{2}, i_{3}}^{(7)} \mu^{2}\left(k \cdot w_{1 ; i_{1}, i_{2}}\right)+c_{i_{1}, i_{2}, i_{3}}^{(8)} \mu^{2}\left(k \cdot w_{2 ; i_{1}, i_{2}}\right)+c_{i_{1}, i_{2}, i_{3}}^{(9)} \mu^{2}, \\
= & c_{i_{1}, i_{2}}^{(0)}+c_{i_{1}, i_{2}}^{(1)}\left(k \cdot w_{1 ; i_{1}}\right)+c_{i_{1}, i_{2}}^{(2)}\left(k \cdot w_{2 ; i_{1}}\right)+c_{i_{1}, i_{2}}^{(3)}\left(k \cdot w_{3 ; i_{1}}\right) \\
& +c_{i_{1}, i_{2}}^{(4)}\left(k \cdot w_{1 ; i_{1}}\right)\left(k \cdot w_{2 ; i_{1}}\right)+c_{i_{1}, i_{2}}^{(5)}\left(k \cdot w_{1 ; i_{1}}\right)\left(k \cdot w_{3 ; i_{1}}\right) \\
& +c_{i_{1}, i_{2}}^{(6)}\left(k \cdot w_{2 ; i_{1}}\right)\left(k \cdot w_{3 ; i_{1}}\right)+c_{i_{1}, i_{2}}^{(7)}\left(\left(k \cdot w_{1 ; i_{1}}\right)^{2}-\left(k \cdot w_{3 ; i_{1}}\right)^{2}\right) \\
& +c_{i_{1}, i_{2}}^{(8)}\left(\left(k \cdot w_{2 ; i_{1}}\right)^{2}-\left(k \cdot w_{3 ; i_{1}}\right)^{2}\right)+c_{i_{1}, i_{2}}^{(9)} \mu^{2} .
\end{aligned}
$$

The irreducible numerators $k \cdot w_{x ; i_{1}, \ldots, i_{s}}$ can be constructed using the spurious directions of van Neerven and Vermaseren [28] and vanish after integration. The spurious directions 
$w_{x ; i_{1}, \ldots, i_{p}}$ are orthogonal to the $p$ dimensional physical space spanned by the momenta entering vertices $i_{1}, \ldots, i_{s}$ where $x=1, \ldots, s$ with $s+p=4 . \mu^{2}=-\tilde{k} \cdot \tilde{k}$ is the extra dimensional scalar product where $k=\bar{k}+\tilde{k}$. These give rise to dimension shifted integrals which in turn lead to rational terms in $d=4-2 \epsilon$ dimensions. The coefficients $c$ are rational functions of the external kinematics and can be extracted from generalised unitarity cuts. After elimination of vanishing integrals over the spurious directions, the $d$-dimensional representation of the amplitude is,

$$
\begin{aligned}
& B_{n}^{(1)}\left(d, d_{s}\right)=\sum_{1 \leq i_{1}<i_{2}<i_{3}<i_{4}<i_{5} \leq n} c_{i_{1}, i_{2}, i_{3}, i_{4}, i_{5}}^{(0)} I_{i_{1}, i_{2}, i_{3}, i_{4}, i_{5}}^{d}\left[\mu^{2}\right] \\
& \quad+\sum_{\substack{1 \leq i_{1}<i_{2}<i_{3}<i_{4} \leq n \\
c_{i_{1}, i_{2}, i_{3}, i_{4}}^{(0)}}} I_{i_{1}, i_{2}, i_{3}, i_{4}}^{d}[1]+c_{i_{1}, i_{2}, i_{3}, i_{4}}^{(2)} I_{i_{1}, i_{2}, i_{3}, i_{4}}^{d}\left[\mu^{2}\right]+c_{i_{1}, i_{2}, i_{3}, i_{4}}^{(4)} I_{i_{1}, i_{2}, i_{3}, i_{4}}^{d}\left[\mu^{4}\right] \\
& +\sum_{1 \leq i_{1}<i_{2}<i_{3} \leq n} c_{i_{1}, i_{2}, i_{3}}^{(0)} I_{i_{1}, i_{2}, i_{3}}^{d}[1]+c_{i_{1}, i_{2}, i_{3}}^{(9)} I_{i_{1}, i_{2}, i_{3}}^{d}\left[\mu^{2}\right] \\
& +\sum_{\substack{1 \leq i_{1}<i_{2} \leq n \\
i_{2}-i_{1} \bmod n>1}} c_{i_{1}, i_{2}}^{(0)} I_{i_{1}, i_{2}}^{d}[1]+c_{i_{1}, i_{2}}^{(9)} I_{i_{1}, i_{2}}^{d}\left[\mu^{2}\right],
\end{aligned}
$$

where

$$
I_{i_{1}, i_{2}, \ldots, i_{n}}^{d}[N]=\mu_{R}^{2 \epsilon} \int \frac{d^{d} k}{(2 \pi)^{d}} \frac{N}{D_{i_{1}} D_{i_{2}} \cdots D_{i_{n}}} .
$$

Explicitly the dimension shifting relations are ${ }^{2}$

$$
\begin{aligned}
& I_{i_{1}, i_{2}, \ldots, i_{n}}^{d}\left[\mu^{2}\right]=\frac{d-4}{2}(4 \pi) I_{i_{1}, i_{2}, \ldots, i_{n}}^{d+2}[1], \\
& I_{i_{1}, i_{2}, \ldots, i_{n}}^{d}\left[\mu^{4}\right]=\frac{(d-4)(d-2)}{4}(4 \pi)^{2} I_{i_{1}, i_{2}, \ldots, i_{n}}^{d+4}[1] .
\end{aligned}
$$

\section{Massive fermion spinors}

In this section we describe how we can use massless six dimensional momenta to obtain amplitudes in four dimensions with massive particles. Before getting started it is helpful to recall how massive fermion wavefunctions can be incorporated within the four-dimensional spinor-helicity formalism commonly used for massless amplitudes. We follow the notation used previously in reference [12] while the formalism itself was established long before that, see for example [29-33].

Starting from a massive 4-momentum $p$ with $p^{2}=m^{2}$, we can define a massless projection with respect to a light-like reference vector $\eta$,

$$
p^{b}=p-\frac{m^{2}}{2 p \cdot \eta} \eta
$$

\footnotetext{
${ }^{2} \mathrm{~A}$ simple derivation of this fact is shown in appendix A.2 of reference [7].
} 
such that $\left(p^{b}\right)^{2}=0$. A complete set of solutions of the Dirac equation for the massive momentum $p$ can then be constructed from the Weyl spinors of $p^{b}$ and $\eta$ :

$$
\begin{array}{ll}
\bar{u}_{+}(p, m)=\frac{\langle\eta|(\not p+m)}{\left\langle\eta p^{b}\right\rangle}, & \bar{u}_{-}(p, m)=\frac{[\eta \mid(\not p+m)}{\left[\eta p^{b}\right]}, \\
v_{+}(p, m)=\frac{(\not p-m)|\eta\rangle}{\left\langle p^{b} \eta\right\rangle}, & v_{-}(p, m)=\frac{(\not p-m) \mid \eta]}{\left[p^{b} \eta\right]} .
\end{array}
$$

These Dirac spinors maintain several of the simplifications which are familiar in the massive case. The tree-level helicity amplitudes for $g g \rightarrow t \bar{t}$ scattering, for example, take the relatively simple forms

$$
\begin{aligned}
& -i\left\langle\eta_{1} 1^{b}\right\rangle\left\langle\eta_{4} 4^{b}\right\rangle A^{(0)}\left(1_{t}^{+}, 2^{+}, 3^{+}, 4_{\bar{t}}^{+}\right)=-\frac{m_{t}^{3} s_{23}\left\langle\eta_{1} \eta_{4}\right\rangle}{2 p_{1} \cdot p_{2}\langle 23\rangle^{2}}, \\
& -i\left\langle\eta_{1} 1^{b}\right\rangle\left\langle\eta_{4} 4^{b}\right\rangle A^{(0)}\left(1_{t}^{+}, 2^{+}, 3^{-}, 4_{\bar{t}}^{+}\right)=\frac{m_{t}\left\langle\eta_{1} 3\right\rangle\left\langle\eta_{4} 3\right\rangle\langle 3|1| 2]}{2 p_{1} \cdot p_{2}\langle 23\rangle}+\frac{m_{t}\left\langle\eta_{1} \eta_{4}\right\rangle\langle 3|1| 2]^{2}}{2 p_{1} \cdot p_{2} s_{23}} .
\end{aligned}
$$

We will now show that these results can be rewritten in terms of amplitudes of massless fermions in six dimensions.

\subsection{Massive fermions from massless six dimensional spinors}

In this section we will use the six dimensional spinor-helicity formalism introduced by Cheung and O'Connell [19] to find representations of four-dimensional massive fermion wave-functions and amplitudes. Further discussion of the six dimensional spinor-helicity formalism can be found in references [20, 21, 34-38]. We begin our discussion by looking at a free massive fermion field in four dimensions,

$$
\mathcal{L}^{4 d}=\bar{\psi}(x)\left(i \gamma^{\mu} \partial_{\mu}-m\right) \psi(x)
$$

In the Weyl representation the Dirac $\gamma$ matrices are

$$
\gamma^{\mu}=\left(\begin{array}{cc}
0 & \tilde{\sigma}^{\mu} \\
\sigma^{\mu} & 0
\end{array}\right),
$$

where $\sigma^{\mu}=\left(1, \sigma^{i}\right), \tilde{\sigma}^{\mu}=\left(1,-\sigma^{i}\right)$ and the usual Pauli matrices, $\sigma^{i}, i=1,2,3$, are written in full in appendix A. For the spinors associated with external fermions we seek solutions to the massive Dirac equation

$$
(\gamma \cdot \bar{p}-m) u_{s}(\bar{p})=0 \text { and } \bar{u}_{s}(\bar{p})(\gamma \cdot \bar{p}-m)=0,
$$

where the bar on the momentum $\bar{p}$ denotes that the vector is in four dimensions.

Alternatively we can consider a massless fermion field in six dimensions, with Lagrangian

$$
\mathcal{L}^{6 d}=\bar{\Psi}(x)\left(i \Gamma^{M} \partial_{M}\right) \Psi(x)
$$


Note that for six dimensions we use capital Greek letters and $M$ runs from 0 to 5 . In six spacetime dimensions the Dirac matrices are $8 \times 8$ objects, which we choose to be

$$
\Gamma^{M}=\left(\begin{array}{cc}
0 & \tilde{\Sigma}^{M} \\
\Sigma^{M} & 0
\end{array}\right),
$$

where the $\Sigma$ matrices are defined by taking outer products of Pauli matrices and are listed explicitly in appendix A. This representation of the $\Gamma$ matrices is simply related to the four dimensional $\gamma$-matrices. The relation for the first four $\Sigma$ matrices is

$$
-\tilde{\Sigma}^{5, A X} \sum_{X B}^{\mu}=\left(\gamma^{\mu}\right)_{B}^{A}=\tilde{\Sigma}^{\mu, A X} \Sigma_{X B}^{5}
$$

where we have adopted the convention that $\Sigma^{M}$ carry lower spinor indices while $\tilde{\Sigma}^{M}$ carry upper indices. For the remaining two $\Sigma$ matrices we have

$$
\begin{aligned}
& -\tilde{\Sigma}^{5, A X} \Sigma_{X B}^{4}=\left(-\gamma^{0} \gamma^{1} \gamma^{2} \gamma^{3}\right)_{B}^{A}=i\left(\gamma^{5}\right)_{B}^{A}, \\
& -\tilde{\Sigma}^{5, A X}{ }_{\Sigma_{X B}^{5}}=\mathbf{1}_{B}^{A} .
\end{aligned}
$$

In the present case, there is no six dimensional mass term. Moreover, in our Weyl basis for the $\Gamma$ matrices (3.9) we can decompose $\Psi=\left(\Psi_{1}, \Psi_{2}\right)$ and see that the two fields decouple:

$$
\mathcal{L}^{6 d}=\bar{\Psi}_{1}(x)\left(i \Sigma^{M} \partial_{M}\right) \Psi_{1}(x)+\bar{\Psi}_{2}(x)\left(i \tilde{\Sigma}^{M} \partial_{M}\right) \Psi_{2}(x) .
$$

Hence the two $\Psi_{i}$ are essentially copies of each other. The spinors associated with these fields each have four components, and the Dirac equation in momentum space associated with $\Psi_{1}(x)$ reads

$$
(\Sigma \cdot p)_{A B} \lambda_{a}^{B}(p)=0
$$

where the $\mathrm{SU}(4)$ indices $A, B$ run from 1 to 4 , and the lower case index, $a$, is a helicity index taking two values. Thus, we may regard the six dimensional spinors as $4 \times 2$ matrices. For the spinors associated with $\Psi_{2}(x)$ the corresponding equation is

$$
(\tilde{\Sigma} \cdot p)^{A B} \tilde{\lambda}_{B \dot{a}}(p)=0 .
$$

We embed the massive four dimensional momentum $\bar{p}$ into a six dimensional massless momentum by declaring that

$$
p=(\bar{p}, 0, m), \text { so } p^{2}=\bar{p}^{2}-m^{2}=0 .
$$

Having made this choice, it is consistent to relate the chiral spinor $\lambda$ to the anti-chiral spinor $\tilde{\lambda}$ by defining

$$
\lambda^{A}=i \tilde{\Sigma}^{4, A B} \tilde{\lambda}_{B}
$$


It is straightforward to verify that this is a solution to the Dirac equation by inserting eq. (3.17) into eq. (3.14) and using the Clifford algebra:

$$
\begin{aligned}
0 & =(\Sigma \cdot p)_{A B} \lambda_{a}^{B}(p) \\
& =i(\Sigma \cdot p)_{A B} \tilde{\Sigma}^{4, B C} \tilde{\lambda}_{C} \\
& =-i \Sigma_{A B}^{4}(\tilde{\Sigma} \cdot p)^{B C} \tilde{\lambda}_{C} \\
\Rightarrow 0 & =(\tilde{\Sigma} \cdot p)^{B C} \tilde{\lambda}_{C} .
\end{aligned}
$$

Having embedded our massive four-dimensional momentum into six dimensions, it is instructive to understand in detail how massless six-dimensional spinors relate to the usual massive four-dimensional Dirac spinors. We begin by writing the massless six-dimensional Dirac equation (3.14) in detail as

$$
(\Sigma \cdot p)_{A B} \lambda_{a}^{B}(p)=\left(\Sigma^{\mu} p_{\mu}-\Sigma^{5} p^{(5)}\right)_{A B} \lambda_{a}^{B}(p)=0 .
$$

Multiplying from the left by $-\tilde{\Sigma}^{5, X A}$ we obtain

$$
\left(\gamma \cdot \bar{p}-p_{1}^{(5)}\right)_{B}^{X} \lambda^{B}(p)=0
$$

Notice how the sign on the sixth component of momentum determines whether $\lambda(p)$ should be associated with the four-dimensional spinor for a fermion $u(p)$ or an anti-fermion $v(p)$ :

$$
\lambda(p)=\left\{\begin{array}{ll}
u(\bar{p}), & p^{(5)}=m \\
v(\bar{p}), & p^{(5)}=-m
\end{array} .\right.
$$

A similar calculation shows how to identify massless six-dimensional spinors with the conjugate four-dimensional Dirac spinors:

$$
\begin{aligned}
0 & =\lambda^{A}(p)\left(\Sigma^{\mu} p_{\mu}-\Sigma^{5} p^{(5)}\right)_{A B} \\
& =\lambda^{A}(p)\left(-\Sigma^{5} \tilde{\Sigma}^{5}\right)_{A}^{X}\left(\Sigma^{\mu} p_{\mu}-\Sigma^{5} p^{(5)}\right)_{X B} \\
& =\lambda^{A}(p) \Sigma_{A X}^{5}\left(\gamma \cdot \bar{p}-p^{(5)}\right)_{B}^{X}
\end{aligned}
$$

Again the sixth momentum component determines whether $\lambda(p) \Sigma^{5}$ should be identified with $\bar{u}(p)$ or $\bar{v}(p)$ :

$$
\lambda(p) \Sigma^{5}=\left\{\begin{array}{ll}
\bar{u}(\bar{p}), & p^{(5)}=m \\
\bar{v}(\bar{p}), & p^{(5)}=-m
\end{array} .\right.
$$

In the following, we find it useful to write an explicit representation for $\lambda^{A}(p)$ that allows us to make direct connection with the specific four-dimensional Dirac spinors given in (3.2). We use a massless (in the four dimensional sense) reference vector $\eta$, as introduced in (3.1), with Weyl spinors $\kappa_{\alpha}(\eta), \tilde{\kappa}^{\dot{\alpha}}(\eta)$ and define the six dimensional spinors:

$$
\lambda^{A a}\left(\eta, \bar{p}^{b}\right)=\left(\begin{array}{cc}
0 & \frac{\tilde{\kappa}^{\dot{\alpha}}(\eta)}{\left[p^{b} \eta\right]} \\
\frac{\kappa_{\alpha}(\eta)}{\left\langle p^{b} \eta\right\rangle} & 0
\end{array}\right), \tilde{\lambda}_{A \dot{a}}\left(\eta, \bar{p}^{b}\right)=\left(\begin{array}{cc}
0 & \frac{\tilde{\kappa}_{\dot{\alpha}}(\eta)}{\left[p^{b} \eta\right]} \\
\frac{\kappa^{\alpha}(\eta)}{\left\langle p^{b} \eta\right\rangle} & 0
\end{array}\right) .
$$


Using $(\Sigma \cdot p)_{A B}(\tilde{\Sigma} \cdot p)^{B C}=0$ we see that the Dirac equation (3.14) is solved by setting

$$
\lambda^{A}(p)=(\tilde{\Sigma} \cdot p)^{A B} \tilde{\lambda}_{B}\left(\eta, \bar{p}^{b}\right) .
$$

The anti-chiral case is completely analogous:

$$
\tilde{\lambda}_{A}(p)=(\Sigma \cdot p)_{A B} \lambda^{B}\left(\eta, \bar{p}^{b}\right) .
$$

The discussion following (3.19) showed how these six dimensional spinors solve the massive Dirac equation in four dimensions with the appropriate choice of sign for $p^{(5)}$.

Since the helicity indices for external particles are not contracted in the six dimensional spinor-helicity formalism, we obtain all helicity configurations in one amplitude. In six dimensions gluons have four polarisation states denoted by two helicity indices. The polarisation vector for a gluon with momentum $p$ is given by

$$
\left.\varepsilon_{a \dot{a}}^{M}=\frac{1}{\sqrt{2}}\left\langle p_{a}\left|\Sigma^{M}\right| q_{b}\right\rangle\left(\left\langle q_{b}\right| p^{\dot{a}}\right]\right)^{-1}
$$

where $q$ is a reference vector satisfying $p \cdot q \neq 0$. Because we use Weyl spinors the fermions still have two states. The amplitude for a quark pair and two gluons is given by [21]

$$
A\left(1_{q, a}, 2_{b \dot{b}}, 3_{c \dot{c}}, 4_{\bar{q}, d}\right)=\frac{-i}{2 s_{12} s_{23}}\left\langle 1_{a} 2_{b} 3_{c} 4_{d}\right\rangle\left[1_{\dot{x}} 2_{\dot{b}} 3_{\dot{c}} 1^{\dot{x}}\right] .
$$

The results in four dimensions are obtained by picking out helicity configurations $(a, b \dot{b}, c \dot{c}, d)=(2,22,22,2),(2,11,22,2)$ for the all plus (3.3) and single minus (3.4) configurations respectively. Other relevant six dimensional trees are given in appendix B.

\subsection{Interactions and state-sum reduction}

We introduce interactions as always by replacing the derivative with the covariant derivative. In six dimensions

$$
\partial_{M} \rightarrow D_{M}=\partial_{M}-i g A_{M}^{i}(x) t^{i}
$$

where $A_{M}^{i}(x)$ are the gauge fields and $t^{i}$ are the generators of the gauge group. We dimensionally reduce the six-dimensional gauge field to four dimensions by treating its last two entries as scalar fields,

$$
A_{M}(x)=A_{M}^{i}(x) t^{i}=\left(A_{\mu}(x), \phi_{1}(x), \phi_{2}(x)\right)
$$

leading to the following interaction terms for $\Psi_{1}$ (dropping dependence on position for simplicity):

$$
\begin{aligned}
\mathcal{L}_{\text {int }, \Psi_{1}}^{6 d} & =-i g \bar{\Psi}_{1} \Sigma^{M} A_{M} \Psi_{1} \\
& =-i g \bar{\Psi}_{1}\left(\Sigma^{\mu} A_{\mu}-\Sigma^{4} \phi_{1}-\Sigma^{5} \phi_{2}\right) \Psi_{1} \\
& =-i g \bar{\Psi}_{1} \Sigma^{\mu} A_{\mu} \Psi_{1}+g \bar{\Psi}_{1} \phi_{1} \Psi_{2}-i g \bar{\Psi}_{1} \phi_{2} \gamma_{5} \Psi_{2},
\end{aligned}
$$




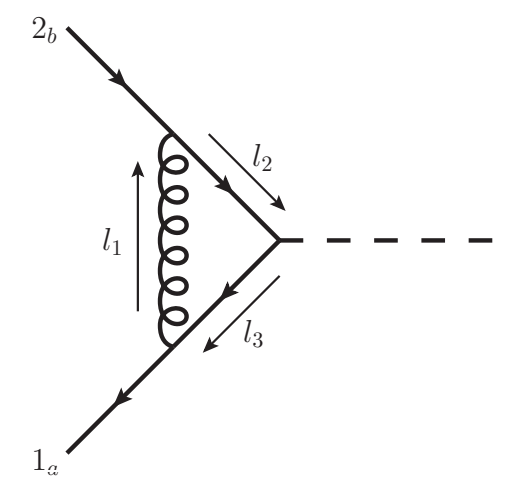

Figure 2. Feynman diagram for one-loop contribution to the coupling between a massive fermion pair and an off-shell scalar. All external momenta are outgoing.

where, in the last line, we have used the relation between chiral and anti-chiral spinors (3.17), which for the fields reads $\Psi_{1}=i \tilde{\Sigma}^{4} \Psi_{2}$. The last two terms give rise to the three-point amplitudes given in (B.8) and (B.9). While the first term resembles the four dimensional interaction term the two last terms are additional contributions arising from the extra momentum components. For internal lines these contributions correspond to additional gluon polarisation states that should be subtracted to obtain the four-dimensional result. This procedure is known as state-sum reduction.

The contraction of Lorentz indices over internal propagators leads to explicit dependence on the spin dimension $d_{s}$. Working explicitly in six dimensions this dependence will be lost but can be recovered through state-sum reduction. The general procedure is described in [21, 27]. Gluons in six dimensions have $6-2=4$ polarisation states, so for each extra dimension introduced we get one more state. Each of these states correspond to the contribution from replacing gluons in the loop by a scalar. By subtracting these scalars the number of polarisation states can be reduced to $d_{s}-2$. In our set-up, the scalar associated with the mass direction should be subtracted separately and we arrive at the state-sum reduction prescription

$$
\boldsymbol{c}=\boldsymbol{c}^{6 d}-\left(5-d_{s}\right) \boldsymbol{c}_{\phi_{1}}-\boldsymbol{c}_{\phi_{2}} .
$$

\subsection{A one-loop example calculation}

Let us now illuminate this higher dimensional formalism with a worked example: the oneloop amplitude for a massive fermion pair coupling to an off-shell scalar, $A^{(1)}$. This calculation involves only one Feynman diagram (figure 2), which, using the colour-ordered four dimensional Feynman rules, is given by

$$
A^{(1), 4 d}=\int \frac{d^{d} \ell_{1}}{(2 \pi)^{d}} \bar{u}_{1} \gamma^{\mu} \frac{\left(\gamma \cdot \ell_{3}+m\right)}{\ell_{3}^{2}-m^{2}} \frac{\left(\gamma \cdot \ell_{2}+m\right)}{\ell_{2}^{2}-m^{2}} \gamma^{\nu} v_{2} \frac{\eta_{\mu \nu}}{\ell_{1}^{2}} \equiv \int \frac{d^{d} \ell_{1}}{(2 \pi)^{d}} \frac{N^{4 d}}{D_{1} D_{2} D_{3}},
$$

where $\ell_{2}=\ell_{1}-p_{2}, \ell_{3}=\ell_{1}+p_{1}, D_{i}=\ell_{i}^{2}-m_{i}^{2}$, and $N^{4 d}$ is the numerator. We will write the result in terms of the scalar integrals using the notation of [39]

$$
\boldsymbol{I}=\left\{I_{3}\left(m^{2}, s, m^{2} ; 0, m^{2}, m^{2}\right), F_{2}\left(s, m^{2}\right), I_{2}\left(m^{2} ; 0, m^{2}\right)\right\}
$$


where $F_{2}\left(s, m^{2}\right)=I_{2}\left(s ; m^{2}, m^{2}\right)-I_{2}\left(m^{2} ; 0, m^{2}\right)$. The result is $A^{(1), 4 d}=\boldsymbol{c}^{\left(d_{s}\right)} \cdot \boldsymbol{I} A^{(0), 4 d}$ where the integral coefficients are given by

$$
\boldsymbol{c}^{\left(d_{s}\right)}=\left\{-2\left(s-2 m^{2}\right),\left(d_{s}-4\right)-\frac{8 m^{2}}{s \beta^{2}}, d_{s}\right\} .
$$

We have set $\beta^{2}=1-\frac{4 m^{2}}{s}$ and $d_{s}$, as usual, is the polarisation state dimension. Using the relation between $\gamma^{\mu}$ and the $\Sigma$ - and $\tilde{\Sigma}$-matrices (3.10) we may simplify the numerator by insertion of $\mathbf{1}_{B}^{A}=-\tilde{\Sigma}^{5, A X} \Sigma_{X B}^{5}$ in (3.33):

$$
\begin{aligned}
N^{4 d} & =\bar{u}_{1} \gamma^{\mu}\left(\gamma \cdot \bar{\ell}_{3}+m\right)\left(\gamma \cdot \bar{\ell}_{2}+m\right) \gamma_{\mu} v_{2} \\
& =\bar{u}_{1} \mathbf{1} \gamma^{\mu}\left(\gamma \cdot \bar{\ell}_{3}+m\right) \mathbf{1}\left(\gamma \cdot \bar{\ell}_{2}+m\right) \mathbf{1} \gamma_{\mu} v_{2} \\
& =-\bar{u}_{1} \tilde{\Sigma}^{5} \Sigma^{\mu}\left(\tilde{\Sigma}^{\nu} \bar{\ell}_{3 \nu}-\tilde{\Sigma}^{5} m\right) \Sigma^{5}\left(\tilde{\Sigma}^{\rho} \bar{\ell}_{2 \rho}-\tilde{\Sigma}^{5} m\right) \Sigma_{\mu} v_{2} \\
& =\lambda_{1} \Sigma^{\mu}\left(\tilde{\Sigma} \cdot \ell_{3}\right) \Sigma^{5}\left(\tilde{\Sigma} \cdot \ell_{2}\right) \Sigma_{\mu} \lambda_{2} .
\end{aligned}
$$

Note the leftover $\Sigma^{5}$ which is associated with the scalar interaction. Hence the tree level amplitude in six dimensions is given by

$$
A^{(0), 6 d}=\lambda_{1} \Sigma^{5} \lambda_{2} .
$$

As we discussed in section 3.2, the contraction of the six-dimensional Lorentz indices of internal gluon lines includes contributions from the extra dimensions. The procedure of reducing the sum over internal states allows us to obtain the explicit dependence on spacetime dimensionality. In the case at hand, the numerator in the six dimensional calculation is:

$$
N^{6 d}=\lambda_{1} \Sigma^{M}\left(\tilde{\Sigma} \cdot \ell_{3}\right) \Sigma^{5}\left(\tilde{\Sigma} \cdot \ell_{2}\right) \Sigma_{M} \lambda_{2}
$$

Comparing with $N^{4 d}$ in equation (3.36), the extra contributions in six dimensions are evidently

$$
\begin{aligned}
& N_{\phi_{1}}^{6 d}=-\lambda_{1} \Sigma^{4}\left(\tilde{\Sigma} \cdot \ell_{3}\right) \Sigma^{5}\left(\tilde{\Sigma} \cdot \ell_{2}\right) \Sigma^{4} \lambda_{2} \\
& N_{\phi_{2}}^{6 d}=-\lambda_{1} \Sigma^{5}\left(\tilde{\Sigma} \cdot \ell_{3}\right) \Sigma^{5}\left(\tilde{\Sigma} \cdot \ell_{2}\right) \Sigma^{5} \lambda_{2} .
\end{aligned}
$$

It follows from (3.31) that contributions from the scalars can equivalently by obtained with

$$
\begin{aligned}
& N_{\phi_{1}}^{6 d}=-\lambda_{1}\left(\Sigma \cdot \ell_{3}\right) \tilde{\Sigma}^{5}\left(\Sigma \cdot \ell_{2}\right) \lambda_{2}, \\
& N_{\phi_{2}}^{6 d}=\lambda_{1} \gamma_{5}\left(\Sigma \cdot \ell_{3}\right) \tilde{\Sigma}^{5}\left(\Sigma \cdot \ell_{2}\right) \tilde{\gamma}_{5} \lambda_{2},
\end{aligned}
$$

where $\left(\tilde{\gamma}_{5}\right)_{B}^{A}=-i \tilde{\Sigma}^{4, A X} \Sigma_{X B}^{5}$. Using the integral basis in (3.34) the result is

$$
\begin{aligned}
A^{(1), 6 d} & =\boldsymbol{c}^{6 d} \cdot \boldsymbol{I} A^{(0), 6 d} \\
& =\left\{-2 s,-\frac{16 m^{2}}{s \beta^{2}}, 4\right\} \cdot \boldsymbol{I} A^{(0), 6 d}, \\
A_{\phi_{1}}^{(1)} & =\boldsymbol{c}_{\phi_{1}} \cdot \boldsymbol{I} A^{(0), 6 d} \\
& =\{0,1,1\} \cdot \boldsymbol{I} A^{(0), 6 d}, \\
A_{\phi_{2}}^{(1)} & =\boldsymbol{c}_{\phi_{2}} \cdot \boldsymbol{I} A^{(0), 6 d} \\
& =\left\{-4 m^{2},-1-\frac{8 m^{2}}{s \beta^{2}},-1\right\} \cdot \boldsymbol{I} A^{(0), 6 d} .
\end{aligned}
$$




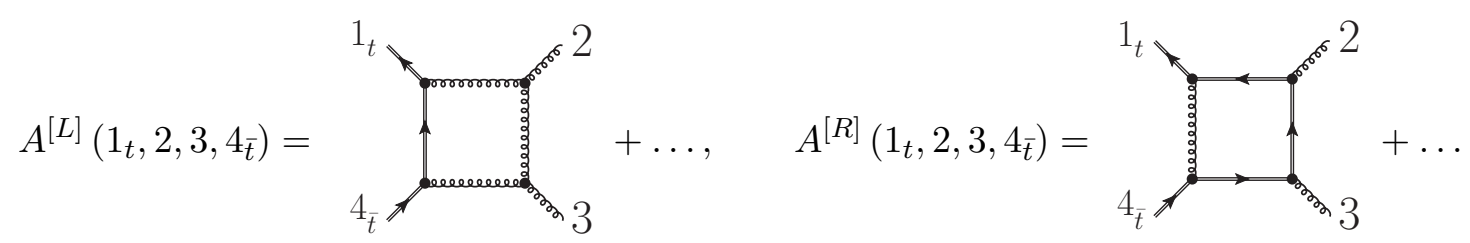

Figure 3. Configurations for left- and right-moving primitive amplitudes contributing to $g g \rightarrow t \bar{t}$ scattering.

The coefficients above are the ingredients needed to perform the state-sum reduction and reproduce (3.35).

\section{Generalised unitarity cuts in six dimensions}

To illustrate our method we consider two gauge invariant primitive amplitudes relevant for the $g g \rightarrow t \bar{t}$ one-loop scattering amplitude. Helicity amplitudes for this process have been previously presented in reference [12]. Using the usual colour decomposition [40] we define the ordered partial amplitudes $A_{4 ; 1}^{(1)}$ and $A_{4 ; 3}^{(1)}$ by,

$$
\mathcal{A}^{(1)}\left(1_{t}, 2,3,4_{\bar{t}}\right)=\sum_{P(2,3)}\left(T^{a_{2}} T^{a_{3}}\right)_{i_{1}}^{\bar{i}_{4}} A_{4 ; 1}^{(1)}\left(1_{t}, 2,3,4_{\bar{t}}\right)+\operatorname{tr}\left(T^{a_{2}} T^{a_{3}}\right) \delta_{i_{1}}^{\bar{i}_{4}} A_{4 ; 3}^{(1)}\left(1_{t}, 4_{\bar{t}} ; 2,3\right),
$$

where $P(2,3)$ is the permutations over the order of gluons. These partial amplitudes can be further decomposed into gauge invariant primitive amplitudes,

$$
\begin{aligned}
A_{4 ; 1}^{(1)}\left(1_{t}, 2,3,4_{\bar{t}}\right)= & N_{c} A^{[L]}\left(1_{t}, 2,3,4_{\bar{t}}\right)-\frac{1}{N_{c}} A^{[R]}\left(1_{t}, 2,3,4_{\bar{t}}\right) \\
& -N_{f} A^{[f]}\left(1_{t}, 2,3,4_{\bar{t}}\right)-N_{H} A^{[H]}\left(1_{t}, 2,3,4_{\bar{t}}\right), \\
A_{4 ; 3}^{(1)}\left(1_{t} 4_{\bar{t}} ; 2,3\right)= & \sum_{P(2,3)}\left(A^{[L]}\left(1_{t}, 2,3,4_{\bar{t}}\right)+A^{[L]}\left(1_{t}, 2,4_{\bar{t}}, 3\right)+A^{[R]}\left(1_{t}, 2,3,4_{\bar{t}}\right)\right),
\end{aligned}
$$

where $N_{c}$ is the number of colours, while $N_{f}$ and $N_{H}$ are the number of light and heavy fermion flavours, respectively. The left-moving $A^{[L]}$ and right-moving $A^{[R]}$ primitive amplitudes are labelled according to the direction of the fermion current as it enters the loop, following the convention of reference [40]. Representative diagrams for these amplitudes are shown in figure 3. We will not consider the fermion loop contributions $A^{[f]}$ and $A^{[H]}$ in this article as they do not present any further technical difficulties.

Each primitive amplitude can be decomposed at the integrand level into the basis of integrals described in section 2. To capture the full $d$-dimensional dependence, we first compute generalised cuts in six dimensions using the spinor-helicity formalism described in the previous section. We then compute the two additional scalar loop contributions and perform the state sum reduction onto a general dimension $d$ according to eq. (3.32). The complete set of generalised cuts needed for the amputated primitives $B^{[L]}$ and $B^{[R]}$, cf. $B_{n}^{(1)}$ in equation (2.1), are shown in figure 4 and 5 , in which the divergent two-particle and one-particle cuts are removed. 

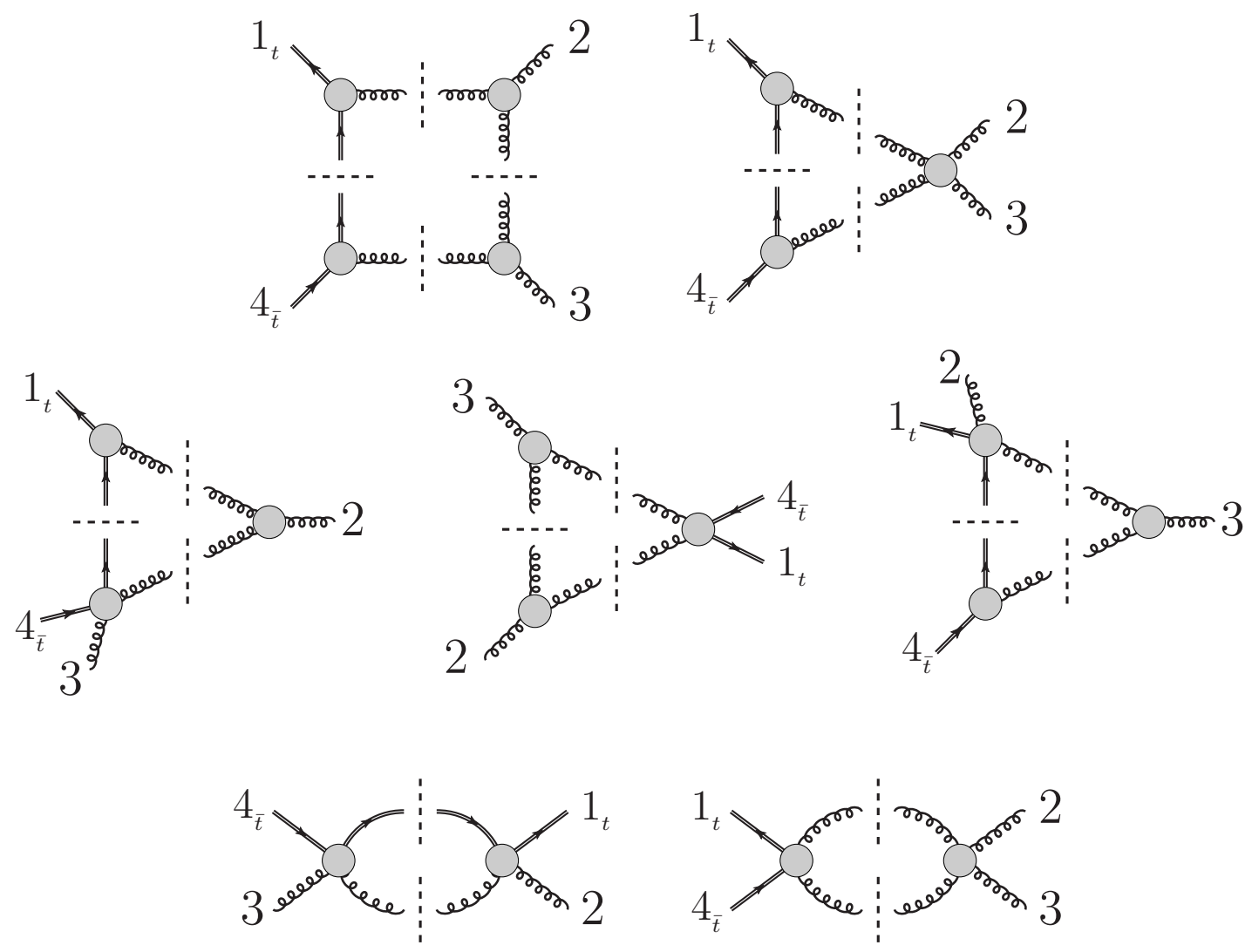

Figure 4. The complete set of cuts for $B^{[L]}\left(1_{t}, 2,3,4_{\bar{t}}\right)$. Double lines represent massive fermions.

Each six-dimensional cut is associated with a set of loop momenta $\ell_{i}$ which enter the tree-level amplitudes. These momenta are determined by solving the system of on-shell equations $\left\{\ell_{i}^{2}=0, i \in S\right\}$. The complete set of loop momenta for our ordered amplitudes are labelled as,

$$
\begin{aligned}
& \ell_{i}^{\mu} \equiv \ell_{0}^{\mu}-P_{i}^{\mu}, \quad P_{i}^{\mu}=\sum_{n=1}^{i} p_{n}^{\mu}, \\
& \ell_{0}^{\mu} \equiv k^{\mu},
\end{aligned}
$$

where $p_{n}^{\mu}$ are the external momenta and $k$ is the loop integration momentum.

The internal particles are embedded into six dimensions by allowing the mass to flow in the sixth component, following our convention in eq. (3.16), and the $(d-4)$ part of the loop momentum to flow in the fifth component,

$$
\begin{aligned}
\text { gluon loop momentum: } & \ell=\{\bar{\ell}, \mu, 0\}, \\
\text { fermion loop momentum: } & \ell=\{\bar{\ell}, \mu, m\} .
\end{aligned}
$$

The gluon and fermion loop propagators can then be expanded into a four-dimensional part and an effective mass term $\mu^{2}$,

$$
\begin{aligned}
\text { gluon propagator: } & \ell^{2}=\bar{\ell}^{2}-\mu^{2}, \\
\text { fermion propagator: } & \ell^{2}=\bar{\ell}^{2}-\mu^{2}-m^{2} .
\end{aligned}
$$



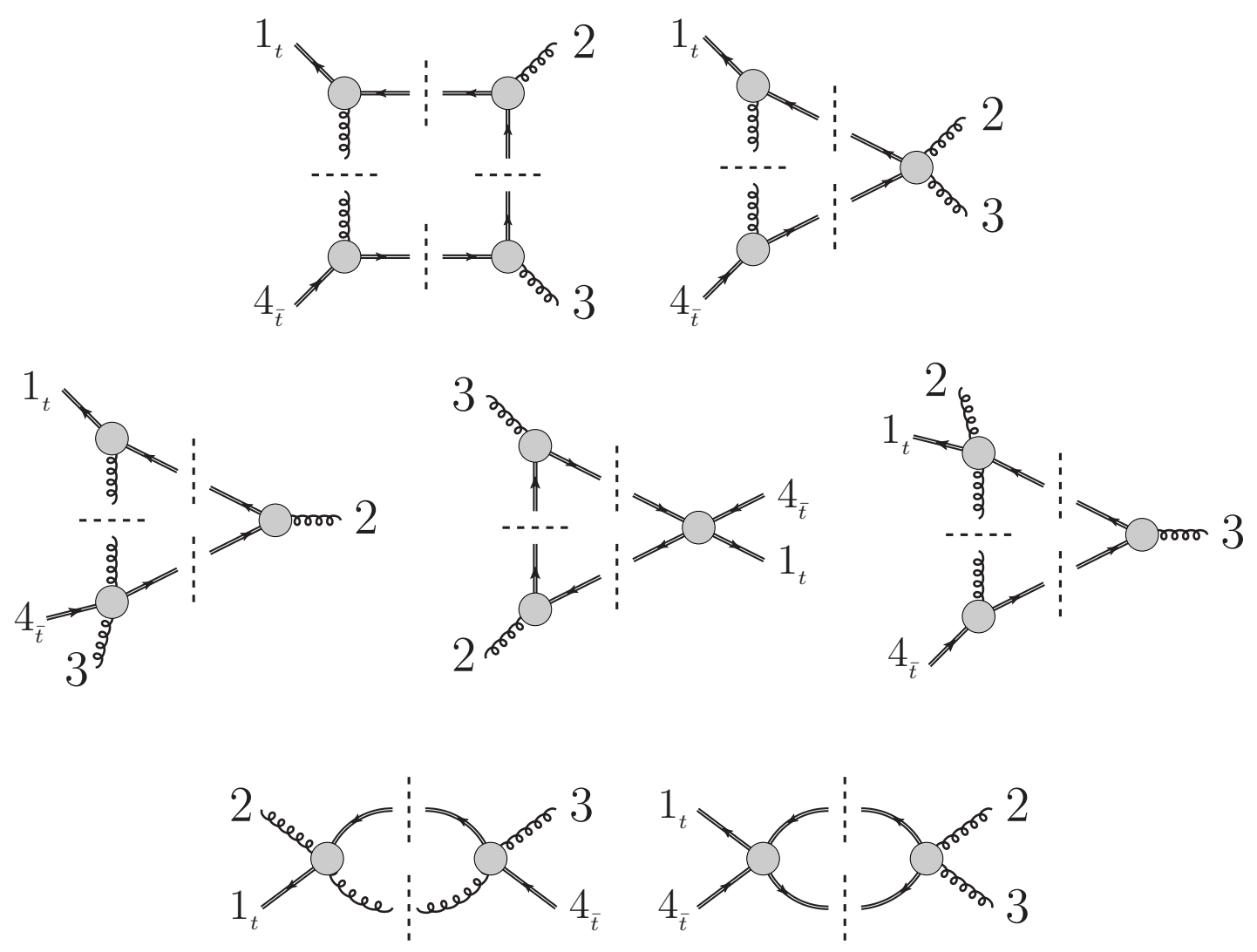

Figure 5. The complete set of cuts for $B^{[R]}\left(1_{t}, 2,3,4_{\bar{t}}\right)$. Double lines represent massive fermions.

This choice is particularly convenient when requiring momentum conservation and orthogonality of the $-2 \epsilon$ component with respect to the external massive fermions momenta expressed in the six dimensional representation, as shown in figure 6.

As an explicit example we will describe the computation of the quadruple cuts. The on-shell equations for these cuts in the left- and right-moving configurations are,

$$
S_{4 ; 0123}^{L}=\left\{\begin{array}{l}
\ell_{0}^{2}=\ell_{1}^{2}=\ell_{2}^{2}=\ell_{3}^{2}=0 \\
\ell_{0}^{(5)}=m
\end{array} \quad, \quad S_{4 ; 0123}^{R}=\left\{\begin{array}{l}
\ell_{0}^{2}=\ell_{1}^{2}=\ell_{2}^{2}=\ell_{3}^{2}=0 \\
\ell_{0}^{(5)}=0
\end{array} .\right.\right.
$$

The constraint on the sixth component of the loop momentum $\ell_{0}$ distinguishes between the two different configurations.

We construct explicit solutions for the six-dimensional spinors of $\ell_{i}$ by introducing arbitrary two-component reference spinors $x_{a}$ and $\tilde{x}_{\dot{a}}$. These solutions, which have a similar form to those presented in refs. [41, 42], take a simple form,

$$
\begin{array}{ll}
\ell_{0}^{M}=\frac{\left\langle x .4\left|\Sigma^{M} 123\right| 4 . \tilde{x}\right]}{\langle x .4|23| 4 . \tilde{x}]}, & \ell_{1}^{M}=\frac{\left\langle x .4\left|1 \tilde{\Sigma}^{M} 23\right| 4 . \tilde{x}\right]}{\langle x .4|23| 4 . \tilde{x}]} \\
\ell_{2}^{M}=\frac{\left\langle x .4\left|12 \Sigma^{M} 3\right| 4 . \tilde{x}\right]}{\langle x .4|23| 4 . \tilde{x}]}, & \ell_{3}^{M}=\frac{\left\langle x .4\left|123 \tilde{\Sigma}^{M}\right| 4 . \tilde{x}\right]}{\langle x .4|23| 4 . \tilde{x}]}
\end{array}
$$




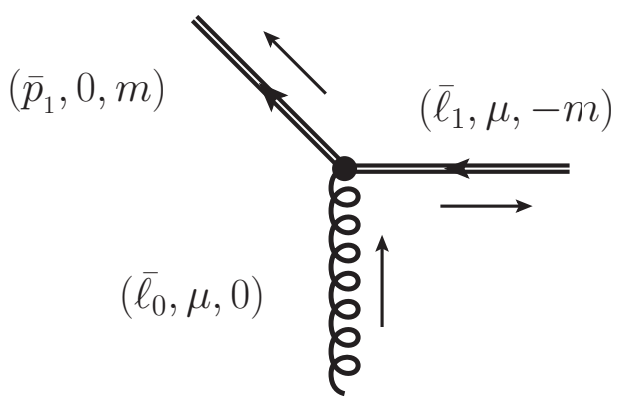

Figure 6. To perform the unitarity cuts of the six dimensional propagators involving internal fermions, we allow the $(d-4)$ part, $\mu$, of the loop momentum to flow in the fifth component and the mass term to flow in the sixth component, in order to easily impose momentum conservation.

where $\left\langle x .4\left|=x^{a}\left\langle 4_{a}|| ,4 . \tilde{x}\right]=\right| 4^{\dot{a}}\right] \tilde{x}_{\dot{a}}$ and the spinor product strings have the following expression (for $n$ even)

$$
\left\langle 1_{a}|23 \ldots(n-1)| n^{\dot{b}}\right]=\lambda_{a}^{A}\left(p_{1}\right)\left(\Sigma \cdot p_{2}\right)_{A B}\left(\tilde{\Sigma} \cdot p_{3}\right)^{B C} \ldots\left(\tilde{\Sigma} \cdot p_{n-1}\right)^{C A} \tilde{\lambda}_{A} \dot{b}^{\dot{b}}\left(p_{n}\right) .
$$

The expressions for the two reference spinors can generically be chosen to be

$$
x_{a}=\left(1, \tau_{1}\right), \quad \tilde{x}_{\dot{a}}=(1, y),
$$

where $y$ is fixed, for left and right, by the mass constraint for $\ell_{0}^{(5)}$ specified in (4.8). Because we have a system of 5 equations for 6 dimensional momenta, the parameter $\tau_{1}$ is left unconstrained.

On the quadruple cut the amplitudes factorise into products of four tree-level amplitudes,

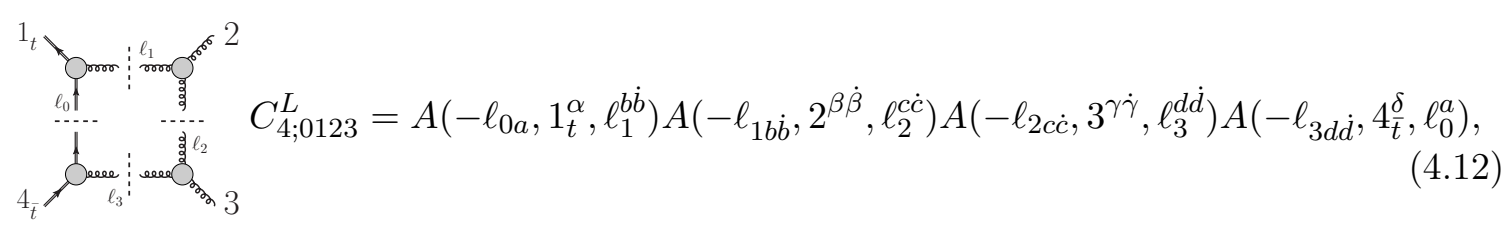

and

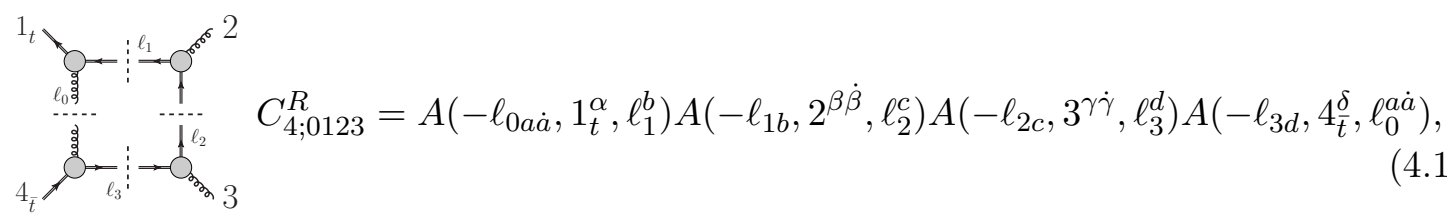

where in both cases the repeated $\mathrm{SU}(2)$ spinor indices are summed over the six dimensional polarisation states.

The integrand reduction method then proceeds to extract the five independent coefficients in the integrand parametrisation from eq. (2.6) by evaluating both the product of trees and the irreducible scalar products $\mu^{2}$ and $k \cdot w_{1 ; 123}$ using the on-shell solution in 
eq. (4.9) and comparing the resulting rational functions in $\tau_{1}$. We encounter an interesting subtlety when following this procedure since the six-dimensional cut contains additional terms which are linear in the extra-dimensional component of the loop momentum $\mu$. These terms are spurious and integrate to zero, but require additional coefficients to be added at the integrand level if this direct approach is taken. A slightly simpler approach is to cancel the linear part of the cut by averaging over the two different flows of the momentum in the fifth component,

$$
\frac{1}{2}\left(\left.C_{4 ; 0123}\right|_{S_{4 ; 0123}^{+}}+\left.C_{4 ; 0123}\right|_{S_{4 ; 0123}^{-}}\right)=\left.\Delta_{\{0,1,2,3\}}\right|_{S_{4 ; 0123}},
$$

where

$$
S^{+}=\left\{\ell_{i}^{2}=0, \ell_{i}=\{\ldots, \mu, \ldots\}\right\}, \quad S^{-}=\left\{\ell_{i}^{2}=0, \ell_{i}=\{\ldots,-\mu, \ldots\}\right\} .
$$

The triangle and bubble coefficients follow using the OPP method to systematically remove all singularities from the cut amplitude using the previously computed irreducible numerators. The mass dependence of the propagators is now dictated by six dimensional momentum conservation applied to the loop momenta, so all propagators are simply $\ell_{i}^{2}$. To remove the terms linear in $\mu$, we average over the two directions for the extra-dimensional component, as described above. Thus,

$$
\begin{aligned}
\left.\frac{1}{2} \sum_{\sigma= \pm} C_{4 ; 0123}\right|_{S_{4 ; 0123}^{\sigma}} & =\left.\Delta_{\{0,1,2,3\}}\right|_{S_{4 ; 0123}}, \\
\left.\frac{1}{2} \sum_{\sigma= \pm} C_{3 ; 012}\right|_{S_{3 ; 012}^{\sigma}}-\left.\frac{\Delta_{\{0,1,2,3\}}}{\ell_{3}^{2}}\right|_{S_{3 ; 012}} & =\left.\Delta_{\{0,1,2\}}\right|_{S_{3 ; 012}}, \\
\left.\frac{1}{2} \sum_{\sigma= \pm} C_{2 ; 02}\right|_{S_{3 ; 02}^{\sigma}}-\left.\left(\frac{\Delta_{\{0,1,2\}}}{\ell_{1}^{2}}+\frac{\Delta_{\{0,2,3\}}}{\ell_{3}^{2}}+\frac{\Delta_{\{0,1,2,3\}}}{\ell_{1}^{2} \ell_{3}^{2}}\right)\right|_{S_{2 ; 02}} & =\left.\Delta_{\{0,2\}}\right|_{S_{2 ; 02}},
\end{aligned}
$$

where the parametrisations for each irreducible numerator are those of equation (2.5). The remaining triple and double cuts follow by permuting the equations (4.16). Further details on the on-shell cut solutions are given in appendix $\mathrm{C}$ and a full set of numerical results for the six dimensional cuts are listed in the MATHEMATICA notebook accompanying this article.

The final step to dimensionally reduce the coefficients from 6 to a general dimension $d$ is to remove the extra degrees of freedom contained in the six dimensional loop momentum according to eq. (3.32). The computation of these extra cuts is done using the same procedure as above, but the internal gluon lines in figures 4 and 5 are replaced with scalar lines. For example, the quadruple cuts are given by the following expressions

$$
\begin{aligned}
& C_{4 ; 0123}^{L, \phi_{(1,2)}}=A\left(-\ell_{0 \dot{a}}, 1_{t}^{\alpha}, \ell_{1}\right) A\left(-\ell_{1}, 2^{\beta \dot{\beta}}, \ell_{2}\right) A\left(-\ell_{2}, 3^{\gamma \dot{\gamma}}, \ell_{3}\right) A\left(-\ell_{3}, 4_{\bar{t}}^{\delta}, \ell_{0}^{\dot{a}}\right), \\
& C_{4 ; 0123}^{R, \phi_{(1,2)}}=A\left(-\ell_{0}, 1_{t}^{\alpha}, \ell_{1}^{\dot{b}}\right) A\left(-\ell_{1 \dot{b}}, 2^{\beta \dot{\beta}}, \ell_{2}^{\dot{c}}\right) A\left(-\ell_{2 \dot{c}}, 3^{\gamma \dot{\gamma}}, \ell_{3}^{\dot{d}}\right) A\left(-\ell_{3 \dot{d}}, 4_{\dot{t}}^{\delta}, \ell_{0}\right) .
\end{aligned}
$$

A complete set of fermion and scalar integrand coefficients are presented in the attached notebook. 


\section{Determining the remaining integral coefficients}

At this point, let us pause to take stock of what has been achieved, and what remains to be done. To do so, we return to equation (2.1), the standard expression for a one-loop amplitude, expanded in a basis of scalar integrals:

$$
A_{n}^{(1)}=B_{n}^{(1)}+c_{2 ; m^{2}} I_{2, m^{2}}+c_{1} I_{1}
$$

By definition, $B_{n}^{(1)}$ is the part of the amplitude which can be computed using finite $d$ dimensional unitarity cuts; its expansion in terms of an integral basis was explicitly given in equation (2.4). We have therefore computed $B_{n}^{(1)}$ explicitly in section 4. A complete construction of the amplitude requires us to find the integral coefficients $c_{2 ; m^{2}}$ and $c_{1}$. This is the task of the present section.

\subsection{Fixing $c_{2, m^{2}}$ by matching the poles in $4-2 \epsilon$ dimensions}

Our first source of additional information is the universal pole structure of four dimensional amplitudes. The poles of general one-loop QCD amplitudes in four dimensions were inferred from the corresponding real-radiation contributions to the NLO cross-section in full generality by Catani, Dittmaier and Trocsanyi [43],

$$
A^{(1), 4-2 \epsilon}=c_{\Gamma} I^{(1)}(\epsilon) A^{(0)}+\text { finite. }
$$

The integrals $I_{2, m^{2}}$ and $I_{1}$ appearing in equation (5.1) are divergent, and therefore the coefficients $c_{2 ; m^{2}}$ and $c_{1}$ contribute to the pole structure of our amplitude. This will allow us to constrain them.

For the simplified case of $t \bar{t}+n(g)$ with $n_{f}$ light quark flavours and one heavy flavour of mass $m$, the function $I^{(1)}(\epsilon)$ appearing in the univeral pole formula is, explicitly,

$$
I^{(1)}(\epsilon)=\frac{n_{g} \beta_{0}\left(n_{f}+1\right)}{2 \epsilon}+\sum_{i, j}\left(\frac{\mu_{R}^{2}}{s_{i j}}\right)^{\epsilon} \mathcal{V}_{i j}-n_{g} \Gamma_{g}-2 \Gamma_{t}+\text { finite. }
$$

Following Catani et al. [43], this formula corresponds to partially renormalised amplitudes. The first term contains UV poles related to charge renormalisation, the second term corresponds to soft-collinear poles and takes the familiar dipole form in colour space. The last terms contain poles given by the anomalous dimensions,

$$
\begin{aligned}
\Gamma_{g} & =\frac{\beta_{0}\left(n_{f}\right)}{2 \epsilon}+\frac{2 T_{R}}{3} \log \left(\frac{\mu_{R}^{2}}{m_{t}^{2}}\right), \\
\Gamma_{t} & =C_{F}\left(\frac{1}{\epsilon}-\frac{1}{2} \log \left(\frac{\mu_{R}^{2}}{m_{t}^{2}}\right)-2\right) .
\end{aligned}
$$

The QCD $\beta$ function appears as a function of the active fermion flavours $\beta_{0}\left(n_{f}\right)=\left(11 C_{A}-\right.$ $\left.4 T_{R} n_{f}\right) / 3$. For the purposes of this paper we will not require the finite parts of $I^{(1)}$ which depend on the dimensional regularisation scheme, e.g. CDR or FDH/DR. The exact form of the function $\mathcal{V}$ is a little more complicated and not of direct relevance here. Clearly there 
is an enormous amount of information contained in this result and further details can be found by consulting the original reference [43].

The simple observation relevant for our approach is that this universal information can be compared to the integral basis in equation (5.1), enabling a partial determination of the unknown coefficients of wavefunction bubble and tadpole integrals. These integrals give rise to single poles in $\epsilon$ and single logarithms in the mass $m$. This comparison is however insufficient to constrain both $c_{2, m^{2}}$ and $c_{1}$.

It is convenient to modify the integral basis slightly, introducing finite bubble and tadpole functions defined by

$$
\begin{aligned}
F_{2 ; i_{1}, i_{2}} & =I_{2, i_{1}, i_{2}}-I_{2, m^{2}}, \\
F_{1} & =I_{1}-m^{2} I_{2, m^{2}} .
\end{aligned}
$$

The result of this modification is that only the finite bubble integrals and the wavefunction integral contribute to the $\log \left(\mu_{R}^{2} / m_{t}^{2}\right)$ dependence of the universal pole structure (5.3). Upon matching the amplitude with the universal pole structure, we find that the amplitude takes the explicit expression

$$
A^{(1)}=\left.A^{6 D,(1)}\right|_{I_{2} \rightarrow F_{2}}+\frac{d_{s}-2}{4} A^{(0)} I_{2, m^{2}}+c_{1} F_{1},
$$

where the only missing information now lies in the tadpole coefficient $c_{1}$.

\subsection{Counterterms for QCD in six dimensions}

Because of our exploitation of the universal four-dimensional pole structure, the one-loop amplitude, in the form given in equation (5.8), has the property that its infrared and ultraviolet poles have been correctly determined. In addition, all logs in the mass $m_{t}$ are correctly reproduced. Indeed, the unknown coefficient $c_{1}$ now multiplies an integral $F_{1}$ which we may explicitly compute:

$$
F_{1} \stackrel{d=\underline{4-}-2 \epsilon}{=}-i c_{\Gamma} m^{2}+\mathcal{O}(\epsilon)=-\frac{i m^{2}}{(4 \pi)^{2}}+\mathcal{O}(\epsilon) .
$$

Since $c_{1}$ is also a rational function, the part of the amplitude which remains to be determined is simply a rational function of the external momenta and masses.

Having made heavy use of higher dimensional methods so far in our computation, it is natural to regard the four-dimensional result we wish to determine as a specialisation of an amplitude that exists in higher dimensions. Indeed, a quantum field theory which is an analogue of $\mathrm{QCD}$ exists in six dimensions. Moreover, in six dimensions the integral $F_{1}$ is no longer simply a finite rational function. It has an epsilon-pole given by

$$
F_{1} \stackrel{d=6-2 \epsilon}{=}-\frac{i m^{4}}{(4 \pi)^{3}} \frac{1}{6 \epsilon}+\mathcal{O}(\epsilon)
$$

We may therefore find $c_{1}$ by comparison with the universal epsilon-pole structure of the amplitude in six dimensions. 
Thus, we are motivated to consider QCD in six dimensions. Above four dimensions QCD ceases to be renormalisable, so to determine the universal epsilon-pole structure in six dimensions we must include higher (mass-)dimension operators ${ }^{3}$ and treat the theory as an effective theory. By power counting, these operators have one or two powers of momentum more than in the usual QCD Lagrangian, so that they have mass-dimension five or six. The point of view we adopt is that the role of the additional operators is simply to provide counterterms, subtracting the infinities from any one-loop amplitude in the theory. Once all the counterterms have been determined, the epsilon-pole structure of any one-loop amplitude is known.

We therefore begin by constructing a basis of the dimension five and six operators which are required for renormalising QCD amplitudes in six dimensions. These operators contain either two quark fields and three derivatives, such as $\mathcal{O}_{1} \equiv i \bar{\psi} \not D \not D \not D \psi$, or are purely bosonic operators such as $\operatorname{tr} F^{\mu \nu} F_{\nu \rho} F^{\rho}{ }_{\mu} \cdot{ }^{4}$ A full list of potential operators appears in table 1.

Since we are only concerned with poles of on-shell amplitudes, rather than of off-shell correlation functions, we need only study operators which lead to independent contributions to the $S$ matrix. It is a well known fact that operators which are related by the classical equations of motion of the theory lead to the same contribution to the $S$ matrix, to all orders of perturbation theory [44-48]. Thus we may simplify the list of operators in table 1 using the equations of motion

$$
\begin{aligned}
i \not D \psi & =m \psi, \\
D^{\mu} F_{\mu \nu}^{a} & =-g \bar{\psi} \gamma^{\nu} T^{a} \psi .
\end{aligned}
$$

It is straightforward to see that many operators in table 1 are related to other operators in our Lagrangian. For example,

$$
\mathcal{O}_{1} \equiv i \bar{\psi} \not D \not D \not D \psi=-i m^{2} \bar{\psi} \not D \psi,
$$

so that $\mathcal{O}_{1}$ does not lead to a new, independent counterterm. It may therefore be omitted.

Our task now is to construct a basis of operators which are independent under the use of the equations of motion, integration by parts etc. To construct such a basis, we consider several categories of operators. Firstly, we will focus on operators containing two quark fields. We classify these operators further according to the powers of derivatives, or of derivatives and field strength insertions as shown in detail in table 1 . We will begin by examining operators containing the largest number of derivatives or field strengths, as the use of the equations of motion may reduce these operators to simpler operators containing fewer derivatives (or field strengths.)

Each of the derivatives contained in operators of type $\left[\bar{\psi} D^{3} \psi\right]$ has one Lorentz index which we must contract using either metric tensors or gamma matrices. By making use of the equations of motion, we may ignore the options of contracting the left-most or rightmost $D$ index against a gamma matrix - such a contraction would reduce to an operator

\footnotetext{
${ }^{3}$ It is linguistically unfortunate that we are now dealing with operators of mass-dimension five and six (using the usual four-dimensional classification of operator dimension) in a theory defined in six spacetime dimensions. We hope that context will make the meaning of the word "dimension" clear.

${ }^{4}$ Recall that a field strength $F$ counts as two derivatives since $\left[D_{\mu}, D_{\nu}\right]=-i g F_{\mu \nu}$.
} 


\begin{tabular}{|c|c|c|}
\hline Quark fields & Operator & Operator class name \\
\hline \multirow{4}{*}{ Two quarks } & $\begin{array}{c}i \bar{\psi} \not D \not D \not D \psi \\
i \bar{\psi} \not D D^{2} \psi \\
i \bar{\psi} D^{\mu} \not D D_{\mu} \psi\end{array}$ & {$\left[\bar{\psi} D^{3} \psi\right]$} \\
\hline & $\begin{array}{c}\bar{\psi} \gamma^{\mu} \gamma^{\nu} F_{\mu \nu} \not D \psi \\
\bar{\psi} D^{\mu} F_{\mu \nu} \gamma^{\nu} \psi \\
\bar{\psi} F_{\mu \nu} \gamma^{\mu} D^{\nu} \psi\end{array}$ & {$[\bar{\psi} D F \psi]$} \\
\hline & $\begin{array}{c}\bar{\psi} \not D \not D \psi \\
\bar{\psi} D^{2} \psi\end{array}$ & {$\left[\bar{\psi} D^{2} \psi\right]$} \\
\hline & $i \bar{\psi} \gamma^{\mu} \gamma^{\nu} F_{\mu \nu} \psi$ & {$[\bar{\psi} F \psi]$} \\
\hline Zero quarks & $\begin{array}{c}i \operatorname{tr} F^{\mu \nu} F_{\nu \rho} F_{\mu}^{\rho} \\
\operatorname{tr} F^{\mu \nu} D^{2} F_{\mu \nu} \\
\operatorname{tr}\left(D^{\mu} F_{\mu \nu}\right)\left(D^{\rho} F_{\rho}{ }^{\nu}\right)\end{array}$ & \\
\hline
\end{tabular}

Table 1. Table of potential higher dimension operators in the 6 dimensional QCD effective Lagrangian. We have ignored four quark operators, which are not relevant for $t \bar{t}+$ gluons scattering at this order, and operators related to those in our table by integration-by-parts or Hermitian conjugation. We have also imposed the parity symmetry of QCD.

with fewer derivatives which we will analyze below. We are left with the unique possibility $\bar{\psi} D^{\mu} \not D D_{\mu} \psi$. However, this operator is equivalent to a linear combination of operators of class $[\bar{\psi} D F \psi]$ and $\left[\bar{\psi} D^{2} \psi\right]$ upon use of the equations of motion, since

$$
\bar{\psi} D^{\mu} \not D D_{\mu} \psi=\bar{\psi}\left(-i m D^{\mu} D_{\mu}-i g D^{\mu} \gamma^{\nu} F_{\mu \nu}\right) \psi .
$$

Therefore, the class $\left[\bar{\psi} D^{3} \psi\right]$ can be completely reduced to simpler operators.

Next, consider the class $[\bar{\psi} D F \psi]$. In this case we again have three possible Lorentz indices which must be contracted against gamma matrices or metric tensors. We may ignore the possibility of contracting the Lorentz index of the covariant derivative against a gamma matrix because of the equations of motion. We are left with two potential operator structures: $\bar{\psi} D^{\mu} F_{\mu \nu} \gamma^{\nu} \psi$ and $\bar{\psi} F_{\mu \nu} D^{\mu} \gamma^{\nu} \psi$. But

$$
\bar{\psi} D^{\mu} F_{\mu \nu} \gamma^{\nu} \psi=\bar{\psi}\left(-g \bar{\psi} \gamma_{\nu} \psi\right) \gamma^{\nu} \psi+\bar{\psi} F_{\mu \nu} D^{\mu} \gamma^{\nu} \psi,
$$

using the Yang-Mills equation. Since we are only interested in processes with two quarks, we will systematically ignore four quark operators. Therefore, we may replace the operator $\bar{\psi} D^{\mu} F_{\mu \nu} \gamma^{\nu} \psi$ with $\bar{\psi} F_{\mu \nu} D^{\mu} \gamma^{\nu} \psi$. This is the only member of the class $[\bar{\psi} D F \psi]$ which is of interest to us.

We now turn to operator structures containing two quark fields but only one extra power of derivatives or gauge fields. Thus the available operator structures are $[\bar{\psi} D D \psi]$ and $[\bar{\psi} F \psi]$. Up to equations of motion, there is only one operator of the first type: $\bar{\psi} D^{\mu} D_{\mu} \psi$. However, this is a reducible operator:

$$
\bar{\psi} D^{\mu} D_{\mu} \psi=\bar{\psi} \not D \not D \psi-\frac{i g}{2} \bar{\psi} F_{\mu \nu} \gamma^{\nu} \gamma^{\mu} \psi
$$


Thus, up to equations of motion, we may reduce the $[\bar{\psi} D D \psi]$ class to the $[\bar{\psi} F \psi]$ class. Because of the antisymmetry of the field strength tensor, there is only one operator in the $[\bar{\psi} F \psi]$ class, namely $\bar{\psi} F_{\mu \nu} \gamma^{\nu} \gamma^{\mu} \psi$.

Finally, we must consider operators containing no quark fields. There are three gauge invariant possibilities: $\operatorname{tr} F^{\mu \nu} F_{\nu \rho} F^{\rho}{ }_{\mu}$, $\operatorname{tr} F_{\mu \nu} D^{2} F^{\mu \nu}$, and $\operatorname{tr}\left(D^{\mu} F_{\mu \nu}\right)\left(D^{\rho} F_{\rho}{ }^{\nu}\right)$. The last of these three operators is equivalent to a four quark operator using the Yang-Mills equation, and is therefore of no interest to us. Meanwhile, the second of the three is equivalent to the other two:

$$
\operatorname{tr} F_{\mu \nu} D^{2} F^{\mu \nu}=-2 \operatorname{tr}\left(D^{\mu} F_{\mu \nu}\right) D_{\alpha} F^{\alpha \nu}-2 i g \operatorname{tr} F_{\nu \mu} F^{\mu}{ }_{\alpha} F^{\alpha \nu} .
$$

As a result, we may also ignore this operator, leaving only $\operatorname{tr} F^{\mu \nu} F_{\nu \rho} F^{\rho}{ }_{\mu}$.

In summary, there are only three higher dimension operators that contribute to the on-shell amplitudes. We may therefore take the full QCD Lagrangian in six dimensions, at one loop order, to be

$$
\begin{aligned}
\mathcal{L}_{\mathrm{QCD}}^{6}=\bar{\psi}(i \not D-m) \psi & -\frac{1}{2} \operatorname{tr} F_{\mu \nu} F^{\mu \nu}+\frac{i}{2} \sigma_{1} g_{s}^{3} m_{t} \bar{\psi} \gamma^{\mu} \gamma^{\nu} F_{\mu \nu} \psi \\
& +i \sigma_{2} g_{s}^{3} \bar{\psi} F_{\mu \nu} \gamma^{\mu} D^{\nu} \psi+\frac{i}{6} \gamma g_{s}^{3} \operatorname{tr}\left(F^{\mu \nu}\left[F_{\mu \lambda}, F_{\nu}{ }^{\lambda}\right]\right)
\end{aligned}
$$

A selection of the resulting Feynman rules are listed in appendix D.

We adopt the point of view that $\sigma_{1}, \sigma_{2}$ and $\gamma$ are couterterms which remove the divergences in loop amplitudes. In addition there are the usual counterterms from the dimension four vertices $t \bar{t} g$ and $g g g$. We can compute the constants $\delta_{t \bar{t} g}, \delta_{g g g}, \sigma_{1}, \sigma_{2}$ and $\gamma$ from simple one-loop vertex graphs. For example, expanding the $t \bar{t} g$ vertex to $\mathcal{O}\left(g_{s}^{3}\right)$ leads to,

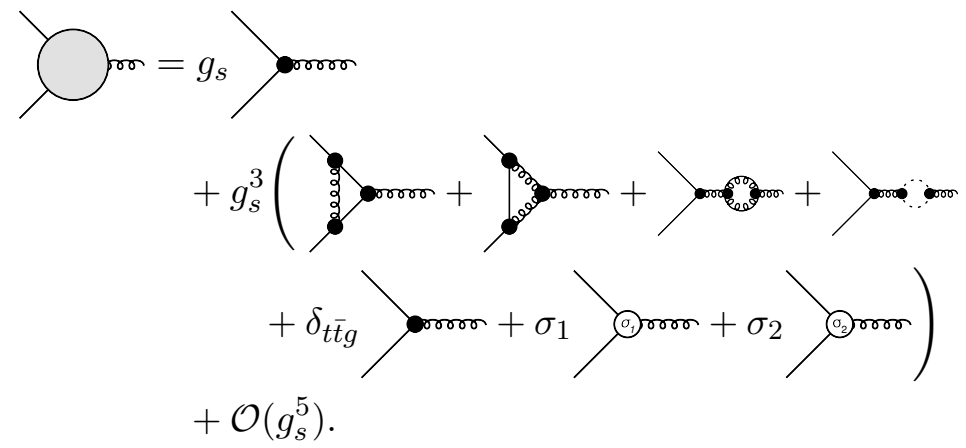

Renormalising this correlation function off-shell would require the inclusion of all possible counterterms (before use of the equations of motion.) For us, it is simpler to compute the on-shell three point vertex, in which case all infinities can be absorbed in our effective Lagrangian, equation (5.18). This presents a minor problem since the three point vertex is not well defined for real momenta. The computation may be performed using complex external kinematics or alternatively performed with the gluon taken off-shell and the constants extracted by taking the on-shell limit $p^{2} \rightarrow 0$ at the end of the computation. We find this amplitude is UV finite in $6-2 \epsilon$ dimensions for the values:

$$
\begin{aligned}
\delta_{t \bar{t} g} & =\frac{m_{t}^{2}}{24(4 \pi)^{3} \epsilon} C_{F}\left(3 d_{s}+2\right), \\
\sigma_{1} & =-\frac{1}{12(4 \pi)^{3} \epsilon}\left(C_{A}\left(d_{s}-5\right)-\frac{C_{F}}{2}\left(3 d_{s}-14\right)\right),
\end{aligned}
$$


where $C_{F}=\frac{N_{c}^{2}-1}{2 N_{c}}$ and $C_{A}=N_{c}$. A similar computation for the three gluon vertex,

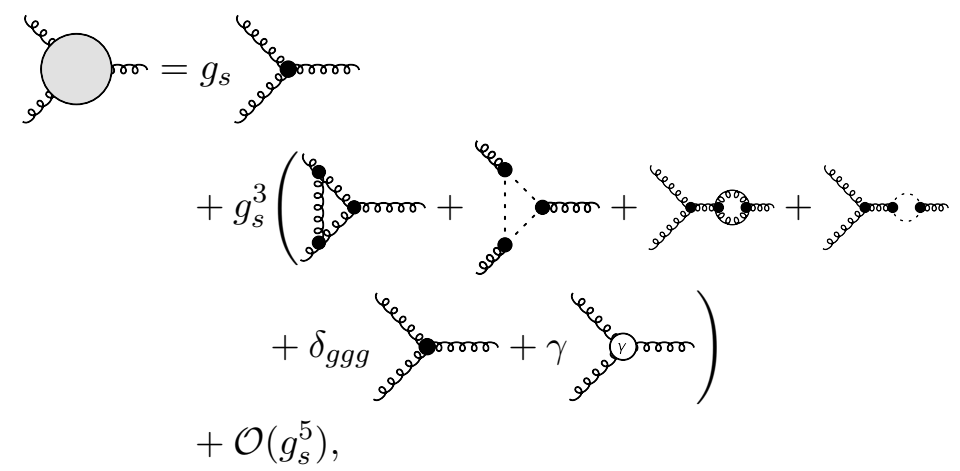

results in

$$
\begin{aligned}
\delta_{g g g} & =0, \\
\gamma & =\frac{1}{12(4 \pi)^{3} \epsilon} C_{A} \frac{\left(d_{s}-2\right)}{5} .
\end{aligned}
$$

\subsection{Fixing $c_{1}$ by matching poles in $6-2 \epsilon$ dimensions}

We finally apply this knowledge of the universal epsilon poles in six dimensions to determine the remaining unknown coefficient, $c_{1}$ in equation (5.8). The six-dimensional leading colour partial amplitude $A_{4 ; 1}^{(1), 6-2 \epsilon}\left(1_{t}, 2,3,4_{\bar{t}}\right)$ can be decomposed into gauge invariant primitives

$$
A_{4 ; 1}^{(1), 6-2 \epsilon}\left(1_{t}, 2,3,4_{\bar{t}}\right)=N_{c} A^{[L], 6-2 \epsilon}\left(1_{t}, 2,3,4_{\bar{t}}\right)-\frac{1}{N_{c}} A^{[R], 6-2 \epsilon}\left(1_{t}, 2,3,4_{\bar{t}}\right),
$$

precisely as in four dimensions (we ignore fermion loops as they present no technical difficulties.) Because the epsilon-poles are universal, we know that the poles of this amplitude are

$$
\begin{aligned}
A_{4 ; 1}^{(1), 6-2 \epsilon}\left(1_{t}, 2,3,4_{\bar{t}}\right)= & g_{s}^{4}\left(2 \delta_{t \bar{t} g} A^{(0)}\left(1_{t}, 2,3,4_{\bar{t}}\right)+\sigma_{1} A^{\left[\sigma_{1}\right]}\left(1_{t}, 2,3,4_{\bar{t}}\right)\right. \\
& \left.+\sigma_{2} A^{\left[\sigma_{2}\right]}\left(1_{t}, 2,3,4_{\bar{t}}\right)+\gamma A^{[\gamma]}\left(1_{t}, 2,3,4_{\bar{t}}\right)\right)+\mathcal{O}\left(\epsilon^{0}\right),
\end{aligned}
$$

where the tree-type amplitudes $A^{\left[\sigma_{1}\right]}\left(1_{t}, 2,3,4_{\bar{t}}\right), A^{\left[\sigma_{2}\right]}\left(1_{t}, 2,3,4_{\bar{t}}\right)$ and $A^{[\gamma]}\left(1_{t}, 2,3,4_{\bar{t}}\right)$ are associated with the three higher dimension operators in the effective 6d QCD Lagrangian, equation (5.18). They are explicitly defined by the diagrams shown in figure 7 . In a similar fashion to the vertex computation we find that $A^{\left[\sigma_{2}\right]}\left(1_{t}, 2,3,4_{\bar{t}}\right)=0$. By collecting in powers of $N_{c}$, and inserting the known expressions for $\delta_{t \bar{t} g}, \sigma_{1}$ and $\gamma$ given in equations (5.20), (5.21) and (5.24) we find,

$$
\begin{gathered}
A^{[L], 6-2 \epsilon}\left(1_{t}, 2,3,4_{\bar{t}}\right)=\frac{g_{s}^{4}}{48(4 \pi)^{3} \epsilon}\left(2\left(3 d_{s}+2\right) m_{t}^{2} A^{(0)}\left(1_{t}, 2,3,4_{\bar{t}}\right)+\frac{4\left(d_{s}-2\right)}{5} A^{[\gamma]}\left(1_{t}, 2,3,4_{\bar{t}}\right)\right. \\
\left.-\left(d_{s}-6\right) A^{\left[\sigma_{1}\right]}\left(1_{t}, 2,3,4_{\bar{t}}\right)\right)+\mathcal{O}\left(\epsilon^{0}\right)
\end{gathered}
$$




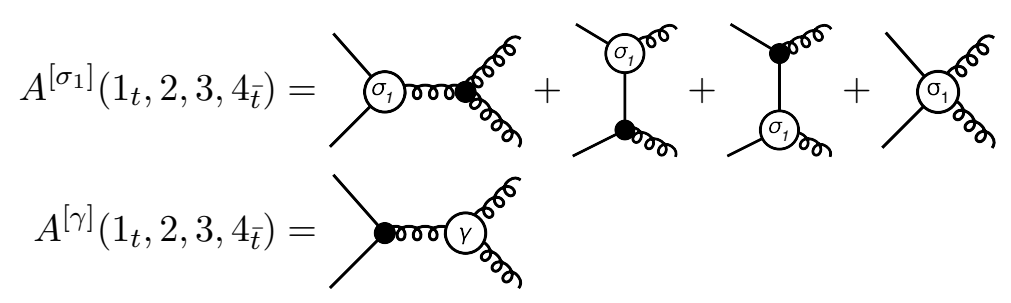

Figure 7. The Feynman diagrams contributing to the tree-level amplitudes appearing the pole structure of the one-loop $t \bar{t} g g$ amplitudes in $6-2 \epsilon$ dimensions. Solid vertices correspond to the usual QCD interactions while the open vertices are those resulting from the corresponding dimension six operators in $\mathcal{L}_{\mathrm{QCD}}^{6}$ of eq. (5.18).

for the left-moving ordering and

$$
\begin{aligned}
A^{[R], 6-2 \epsilon}\left(1_{t}, 2,3,4_{\bar{t}}\right)=\frac{g_{s}^{4}}{48(4 \pi)^{3} \epsilon}( & 2\left(3 d_{s}+2\right) m_{t}^{2} A^{(0)}\left(1_{t}, 2,3,4_{\bar{t}}\right) \\
& \left.+\left(3 d_{s}-14\right) A^{\left[\sigma_{1}\right]}\left(1_{t}, 2,3,4_{\bar{t}}\right)\right)+\mathcal{O}\left(\epsilon^{0}\right)
\end{aligned}
$$

for the right-moving case.

The tree amplitudes $A^{\left[\sigma_{1}\right]}\left(1_{t}, 2,3,4_{\bar{t}}\right)$ and $A^{[\gamma]}\left(1_{t}, 2,3,4_{\bar{t}}\right)$ are easily determined by calculating the diagrams in figure 7 . Written in terms of four dimensional spinor products, the independent helicity amplitudes are

$$
\begin{aligned}
& -i\left\langle\eta_{1} 1^{b}\right\rangle\left\langle\eta_{4} 4^{b}\right\rangle A^{\left[\sigma_{1}\right]}\left(1_{t}^{+}, 2^{+}, 3^{+}, 4_{\bar{t}}^{+}\right)= \\
& \quad \frac{-2 m_{t}\left(2 m_{t}^{2}-4 p_{1} \cdot p_{2}-s_{23}\right) s_{23}\left\langle\eta_{1} 2\right\rangle\left\langle\eta_{4} 3\right\rangle}{\langle 23\rangle^{3}}+\frac{2 m_{t}\left(m_{t}^{2}-2 p_{1} \cdot p_{2}\right) s_{23}\left\langle\eta_{1} \eta_{4}\right\rangle}{m_{t}\langle 23\rangle^{2}} \\
& \quad-\frac{m_{t}\left(m_{t}^{2}-2 p_{1} \cdot p_{2}\right) s_{23}\left\langle\eta_{1} 3\right\rangle\left\langle\eta_{4} 3\right\rangle\langle 2|1| 3]}{p_{1} \cdot p_{2}\langle 23\rangle^{3}}+\frac{m_{t}\left(m_{t}^{2}-2 p_{1} \cdot p_{2}\right) s_{23}\left\langle\eta_{1} 2\right\rangle\left\langle\eta_{4} 2\right\rangle\langle 3|1| 2]}{p_{1} \cdot p_{2}\langle 23\rangle^{3}} \\
& -i\left\langle\eta_{1} 1^{b}\right\rangle\left\langle\eta_{4} 4^{b}\right\rangle A^{\left[\sigma_{1}\right]}\left(1_{t}^{+}, 2^{+}, 3^{-}, 4_{\bar{t}}^{+}\right)=\frac{\left(-4 m_{t}\left(p_{1} \cdot p_{2}\right)^{2}+m_{t}^{2} s_{23}-2 p_{1} \cdot p_{2} s_{23}\right)\left\langle\eta_{1} 3\right\rangle\left\langle\eta_{4} 3\right\rangle\langle 3|1| 2]}{p_{1} \cdot p_{2} s_{23}\langle 23\rangle} \\
& -\frac{2 m_{t}\left\langle\eta_{1} \eta_{4}\right\rangle\langle 3|1| 2]^{2}}{m_{t} s_{23}}+\frac{m_{t}\left(4 p_{1} \cdot p_{2}+s_{23}\right)\left\langle\eta_{1} 2\right\rangle\left\langle\eta_{4} 3\right\rangle\langle 3|1| 2]^{2}}{p_{1} \cdot p_{2} s_{23}\langle 23\rangle}-\frac{m_{t}\left\langle\eta_{1} 2\right\rangle\left\langle\eta_{4} 2\right\rangle\langle 3|1| 2]^{3}}{p_{1} \cdot p_{2} s_{23}\langle 23\rangle} \\
& -i\left\langle\eta_{1} 1^{b}\right\rangle\left\langle\eta_{4} 4^{b}\right\rangle A^{[\gamma]}\left(1_{t}^{+}, 2^{+}, 3^{+}, 4_{\bar{t}}^{+}\right)=\frac{m_{t} s_{23}^{2}\left\langle\eta_{1} 2\right\rangle\left\langle\eta_{4} 3\right\rangle}{2\langle 23\rangle^{3}}+\frac{m_{t} p_{1} \cdot p_{2} s_{23}\left\langle\eta_{1} \eta_{4}\right\rangle}{\langle 23\rangle^{2}} \\
& -i\left\langle\eta_{1} 1^{b}\right\rangle\left\langle\eta_{4} 4^{b}\right\rangle A^{[\gamma]}\left(1_{t}^{+}, 2^{+}, 3^{-}, 4_{\bar{t}}^{+}\right)=0 \\
& -i\left\langle\eta_{1} 1^{b}\right\rangle\left\langle\eta_{4} 4^{b}\right\rangle A^{\left[\sigma_{2}\right]}\left(1_{t}^{+}, 2^{+}, 3^{+}, 4_{\bar{t}}^{+}\right)=0 \\
& -i\left\langle\eta_{1} 1^{b}\right\rangle\left\langle\eta_{4} 4^{b}\right\rangle A^{\left[\sigma_{2}\right]}\left(1_{t}^{+}, 2^{+}, 3^{-}, 4_{\bar{t}}^{+}\right)=0 .
\end{aligned}
$$

We note that the amplitudes of the $\sigma_{2}$ operator vanish in the cases we have considered. We still include it in our analysis since the operator remains in the Lagrangian after using the equations of motion despite not playing a role for the amplitudes in this paper. 
The final step necessary to determine the tadpole coefficient is to evaluate the poles of the basis integrals of the one-loop amplitude in $6-2 \epsilon$ dimensions. We find

$$
\begin{aligned}
I_{1}^{6-2 \epsilon}[1]\left(m^{2}\right) & =\frac{-i m^{4}}{2(4 \pi)^{3} \epsilon}+\mathcal{O}\left(\epsilon^{0}\right) \\
I_{2}^{6-2 \epsilon}[1]\left(P^{2}, m_{1}^{2}, m_{2}^{2}\right) & =i \frac{P^{2}-3\left(m_{1}^{2}+m_{2}^{2}\right)}{6(4 \pi)^{3} \epsilon}+\mathcal{O}\left(\epsilon^{0}\right) \\
I_{2}^{6-2 \epsilon}\left[\mu^{2}\right]\left(P^{2}, m_{1}^{2}, m_{2}^{2}\right) & =i \frac{P^{4}-5 P^{2}\left(m_{1}^{2}+m_{2}^{2}\right)+10\left(\left(m_{1}^{2}+m_{2}^{2}\right)^{2}-m_{1}^{2} m_{2}^{2}\right)}{60(4 \pi)^{3} \epsilon}+\mathcal{O}\left(\epsilon^{0}\right), \\
I_{3}^{6-2 \epsilon}[1] & =\frac{-i}{2(4 \pi)^{3} \epsilon}+\mathcal{O}\left(\epsilon^{0}\right) \\
I_{3}^{6-2 \epsilon}\left[\mu^{2}\right]\left(P_{1}^{2}, P_{2}^{2}, P_{3}^{2}, m_{1}^{2}, m_{2}^{2}, m_{3}^{2}\right) & =-i \frac{P_{1}^{2}+P_{2}^{2}+P_{3}^{2}-4\left(m_{1}^{2}+m_{2}^{2}+m_{3}^{2}\right)}{24(4 \pi)^{3} \epsilon}+\mathcal{O}\left(\epsilon^{0}\right), \\
I_{4}^{6-2 \epsilon}[1] & =\mathcal{O}\left(\epsilon^{0}\right), \\
I_{4}^{6-2 \epsilon}\left[\mu^{2}\right] & =\frac{i}{6(4 \pi)^{3} \epsilon}+\mathcal{O}\left(\epsilon^{0}\right) .
\end{aligned}
$$

We do not list the formulae for box integrals in 10 dimensions $\left(\mu^{4}\right)$ since they do not appear in amplitudes with a fermion pair and any number of gluons. The formulae are easy to derive using the dimensional recurrence relation implemented in LITERED [49] in any case.

The only unknowns in equations (5.27) and (5.28) are then the left- and right-moving tadpole coefficients $c_{1}$, allowing a direct determination of these rational functions. The results are somewhat lengthy formulae which are explicitly derived in the MATHEMATICA workbook. We have checked that this procedure matches the expected result by comparing with the previous computation of reference [12].

\section{Conclusions}

In this paper we have explored a new technique for the computation of one-loop amplitudes with massive fermions. Our methods are designed to be compatible with on-shell generalised unitarity.

The six-dimensional spinor-helicity scheme proved to be an efficient way to describe the tree-level input into the $d$-dimensional generalised unitarity method. Divergent wavefunction cuts were avoided, and the remaining ambiguities in the amplitudes were fixed by matching to the universal physical pole structure. The $4-2 \epsilon$ pole structure of Catani et al. [43] is sufficient to constrain all remaining logarithms in the fermion mass while additional information is needed to fix the remaining finite corrections connected to tadpole integrals. We obtained this second constraint by allowing the loop momenta in our integrals to be defined in a higher dimension spacetime, and imposing the universality of ultraviolet divergences in this higher dimensional quantum field theory. Since six is the next even dimension above four it was natural to study QCD as an effective theory in $6-2 \epsilon$ dimensions. We used the on-shell equations of motion to find a minimal set of additional dimension six operators in this theory, and computed the required counterterms essentially following the 
textbooks. We applied our method to a variety of simple cases and validated it on helicity amplitudes for top quark pair production.

The methods we used in this paper are flexible, and it is clear that they apply more generally than to $g g \rightarrow t \bar{t}$ scattering. It would be interesting to work out the extension to more general cases with multiple fermions and multiple masses, as well as to higher loops. In the presence of more fermions, four quark operators would need to be included in the effective Lagrangian, while at higher loops one would need to consider operators of mass-dimension greater than six.

Since this method can compute amplitudes with fewer cuts than other known approaches it has the potential to optimise existing numerical and analytical approaches. However, since the main computational bottleneck in most phenomenological collider studies at NLO lies in the integration over the unresolved phase-space, the technique is probably best suited to find compact analytic expressions where the improvement in stability and speed over existing numerical approaches is particularly beneficial.

Perhaps a more interesting direction would be to look into the implications of the higher dimensional pole structure on the spurious singularities appearing in integral reductions. As a result of matching to a tree-level computation with an effective Lagrangian, we find non-trivial relations between the $d$-dimensional integral coefficients in which all spurious poles cancel. These cancellations had to occur, since the effective theory contains only local operators. This information could be useful in finding compact and stable representations of one-loop amplitudes.

\section{Acknowledgments}

We would like to thank Fabrizio Caola, Richard Ball, Einan Gardi and Kirill Melnikov for useful discussions. SB is supported by an STFC Rutherford Fellowship ST/L004925/1 and CBH is supported by Rutherford Grant ST/M004104/1. DOC would like to thank the Urriðakvísl 25 Foundation for hospitality during completion of this work. DOC is an IPPP associate, and thanks the IPPP for on-going support as well as for hospitality during this work. This research was supported in part by the European Union through the ERC Advanced Grant MC@NNLO (340983).

\section{A Conventions and spinor construction}

We use the mostly minus metrics

$$
\begin{aligned}
\eta^{\mu \nu} & =\operatorname{diag}\{1,-1,-1,-1\}, \\
\eta^{M N} & =\operatorname{diag}\{1,-1,-1,-1,-1,-1\},
\end{aligned}
$$

where lower-case Greek letters are used for four dimensional Lorentz indices and upper-case for six dimensions. 
We use the following Pauli matrices:

$$
\sigma_{\alpha \dot{\alpha}}^{1}=\left(\begin{array}{cc}
0 & -1 \\
-1 & 0
\end{array}\right), \sigma_{\alpha \dot{\alpha}}^{2}=\left(\begin{array}{cc}
0 & i \\
-i & 0
\end{array}\right), \sigma_{\alpha \dot{\alpha}}^{3}=\left(\begin{array}{cc}
-1 & 0 \\
0 & 1
\end{array}\right),
$$

and $\left(\tilde{\sigma}^{\mu}\right)^{\dot{\alpha} \alpha}=\epsilon^{\alpha \beta} \epsilon^{\dot{\alpha} \dot{\beta}} \sigma_{\beta \dot{\beta}}^{\mu}$ with $\epsilon_{12}=1$. Using the Pauli matrices we define the $\Sigma$-matrices

$$
\begin{array}{ll}
\Sigma^{0}=i \sigma^{1} \times \sigma^{2} & \tilde{\Sigma}^{0}=-i \sigma^{1} \times \sigma^{2} \\
\Sigma^{1}=i \sigma^{2} \times \sigma^{3} & \tilde{\Sigma}^{1}=i \sigma^{2} \times \sigma^{3} \\
\Sigma^{2}=\sigma^{2} \times \sigma^{0} & \tilde{\Sigma}^{2}=-\sigma^{2} \times \sigma^{0} \\
\Sigma^{3}=-i \sigma^{2} \times \sigma^{1} & \tilde{\Sigma}^{3}=-i \sigma^{2} \times \sigma^{1} \\
\Sigma^{4}=-\sigma^{3} \times \sigma^{2} & \tilde{\Sigma}^{4}=\sigma^{3} \times \sigma^{2} \\
\Sigma^{5}=-i \sigma^{0} \times \sigma^{2} & \tilde{\Sigma}^{5}=-i \sigma^{0} \times \sigma^{2},
\end{array}
$$

which obey the Clifford algebra

$$
\Sigma^{M} \tilde{\Sigma}^{N}+\Sigma^{N} \tilde{\Sigma}^{M}=2 \eta^{M N} .
$$

\section{B Tree-level amplitudes in six dimensions}

In this section we list the tree-level amplitudes in six dimensions used in our calculation.

\section{B.1 Three-point amplitudes}

- $A^{(0)}\left(1_{q}, 2_{\bar{q}}, 3_{g}\right)$

$$
\left.A^{(0)}\left(1_{q}^{a}, 2_{\bar{q}}^{b}, 3_{g}^{c \dot{c}}\right)=\frac{i}{s_{r 3}}\left\langle 1^{a} 2^{b} 3^{c} r^{x}\right\rangle\left\langle r_{x}\right| 3^{\dot{c}}\right]
$$

where $r$ is a massless reference vector satisfying $s_{r 3} \neq 0$.

- $A^{(0)}\left(1_{g}, 2_{g}, 3_{g}\right)$

$$
\begin{array}{ll}
A^{(0)}\left(1_{a \dot{a}}, 2_{b \dot{b}}, 3_{c \dot{c}}\right)=i \Gamma_{a b c} \tilde{\Gamma}_{\dot{a} \dot{b} \dot{c}} & \Gamma_{a b c}=u_{1 a} u_{2 b} w_{3 c}+u_{1 a} w_{2 b} u_{3 c}+w_{1 a} u_{2 b} u_{3 c} \\
& \tilde{\Gamma}_{\dot{a} \dot{b} \dot{c}}=\tilde{u}_{1 \dot{a}} \tilde{u}_{2 \dot{a}} \tilde{w}_{3 \dot{c}}+\tilde{u}_{1 \dot{a}} \tilde{w}_{2 \dot{a}} \tilde{u}_{3 \dot{c}}+\tilde{w}_{1 \dot{a}} \tilde{u}_{2 \dot{a}} \tilde{u}_{3 \dot{c}}
\end{array}
$$

where the tensors $\Gamma$ and $\tilde{\Gamma}$ are written in terms of the $\mathrm{SU}(2)$ spinors $u, \tilde{u}$ satisfying the following properties, defined on a cyclic order $\{i j k\}$,

$$
\left.\left.u_{i a} \tilde{u}_{j \dot{b}}=\left\langle i_{a}\right| j_{\dot{b}}\right], \quad \quad u_{j a} \tilde{u}_{i \dot{b}}=-\left\langle j_{a}\right| \dot{i}_{\dot{b}}\right],
$$

and $w, \tilde{w}$ are the inverse of the $u, \tilde{u}$

$$
\epsilon_{a b}=u_{a} w_{b}-u_{b} w_{a}, \quad \epsilon_{\dot{a} \dot{b}}=\tilde{u}_{\dot{a}} \tilde{w}_{\dot{b}}-\tilde{u}_{\dot{b}} \tilde{w}_{\dot{a}},
$$

for which we impose momentum conservation

$$
0=\tilde{w}_{1 \dot{a}} \tilde{\lambda}_{1 A}^{\dot{a}}+\tilde{w}_{2 \dot{a}} \tilde{\lambda}_{2 A}^{\dot{a}}+\tilde{w}_{3 \dot{a}} \tilde{\lambda}_{3 A}^{\dot{a}} .
$$


- $A^{(0)}\left(1_{\phi_{1,2}}, 2_{\phi_{1,2}}, 3_{g}\right)$

$$
\left.A^{(0)}\left(1_{\phi_{1,2}}, 2_{\phi_{1,2}}, 3_{g}^{a \dot{a}}\right)=\frac{-i}{2 s_{r 3}}\left\langle 3^{a}|(1-2)| r\right| 3^{\dot{a}}\right]
$$

where $r$ is a massless reference vector satisfying $s_{r 3} \neq 0$.

- $A^{(0)}\left(1_{\phi_{1}}, 2_{\bar{q}}, 3_{q}\right)$

$$
\left.A^{(0)}\left(1_{\phi_{1}}, 2_{\bar{q}}^{a}, 3_{q}^{\dot{b}}\right)=\frac{i}{\sqrt{2}}\left\langle 1^{a}\right| 2^{\dot{b}}\right]
$$

- $A^{(0)}\left(1_{\phi_{2}}, 2_{\bar{q}}, 3_{q}\right)$

$$
A^{(0)}\left(1_{\phi_{1}}, 2_{\bar{q}}^{a}, 3_{q}^{\dot{b}}\right)=\frac{i}{\sqrt{2}}\left\langle 1^{a}\left|\gamma^{5}\right| 2^{\dot{b}}\right]
$$

\section{B.2 Four-point amplitudes}

- $A^{(0)}\left(1_{g}, 2_{g}, 3_{g}, 4_{g}\right)$

$$
A^{(0)}\left(1_{a \dot{a}}, 2_{b \dot{b}}, 3_{c \dot{c}}, 4_{d \dot{d}}\right)=\frac{-i}{s_{12} s_{23}}\left\langle 1_{a} 2_{b} 3_{c} 4_{d}\right\rangle\left[1_{\dot{a}} 2_{\dot{b}} 3_{\dot{c}} 4_{\dot{d}}\right]
$$

- $A\left(1_{q}, 2_{g}, 3_{g}, 4_{\bar{q}}\right)$

$$
A^{(0)}\left(1_{q, a}, 2_{b \dot{b}}, 3_{c \dot{c}}, 4_{\bar{q}, d}\right)=\frac{i}{2 s_{12} s_{23}}\left\langle 1_{a} 2_{b} 3_{c} 4_{d}\right\rangle\left[1_{\dot{x}} 2_{\dot{b}} 3_{\dot{c}} 1^{\dot{x}}\right] .
$$

- $A^{(0)}\left(1_{g}, 2_{g}, 3_{\phi_{1,2}}, 4_{\phi_{1,2}}\right)$

$$
A^{(0)}\left(1_{a \dot{a}}, 2_{b \dot{b}}, 3,4\right)=\frac{i}{4 s_{12} s_{23}}\left\langle 1_{a} 2_{b} 3_{x} 3^{x}\right\rangle\left[1_{\dot{a}} 2_{\dot{b}} 3_{\dot{x}} 3^{\dot{x}}\right]
$$

\section{Cut solutions in six dimensions}

In this section we give details on the solutions for the triple- and double-cuts in six dimensions. We will describe the parametrisation used to get the solutions without writing down any explicit expression for them. The implementation is given in the attached MATHEMATICA notebook. Notice that all the cut solutions are rational functions of the kinematics and the free parameters and contain no square roots.

\section{C.1 Triple cut}

We write the loop momentum $\ell_{i}^{\mu}$ in the following basis,

$$
\beta=\left\{v^{\mu}, w^{\mu},\left\langle v^{1}\left|\Sigma^{\mu}\right| w_{1}\right\rangle,\left\langle v^{1}\left|\Sigma^{\mu}\right| w_{2}\right\rangle,\left\langle v^{2}\left|\Sigma^{\mu}\right| w_{1}\right\rangle,\left\langle v^{2}\left|\Sigma^{\mu}\right| w_{2}\right\rangle\right\},
$$

where $v$ and $w$ are six dimensional massless momenta and use the parametrisation

$$
\ell_{i}=\beta \cdot\left\{y_{1}, y_{2}, y_{3}, y_{4}, \tau_{1}, \tau_{2}\right\}
$$


We impose the cut conditions

$$
S_{i j k}=\left\{\begin{array}{l}
\ell_{i}^{2}=\ell_{j}^{2}=\ell_{k}^{2}=0 \\
\ell_{i}^{(5)}= \begin{cases}0 & \text { if } i \text { gluon } \\
\pm m & \text { if } i \text { fermion }\end{cases}
\end{array}\right.
$$

where $\{i j k\}$ is the set of the three cut propagators and the sign of the mass component depends on the kinematic configuration. This system of equations for $\ell_{i}$ only constrains 4 parameters so solving for the $y_{i}{ }^{\prime} \mathrm{s}, \tau_{1}, \tau_{2}$ are left as free parameters.

\section{C.2 Double cut}

For the double cut solutions we use the basis in (C.1) and use the following parametrisation

$$
\ell_{i}=\beta \cdot\left\{y_{1}, \tau_{1}, y_{2}, \tau_{2}, y_{3}, \tau_{3}\right\}
$$

The $y_{i}$ 's are fixed by the double cut constraints

$$
S_{i j}=\left\{\begin{array}{l}
\ell_{i}^{2}=\ell_{j}^{2}=0 \\
\ell_{i}^{(5)}= \begin{cases}0 & \text { if } i \text { gluon } \\
\pm m & \text { if } i \text { fermion }\end{cases}
\end{array}\right.
$$

where $\{i j\}$ is the set of the two cut propagators and the sign of the mass component depends on the kinematic configuration. The parameters $\tau_{1}, \tau_{2}, \tau_{3}$ are unconstrained.

\section{Feynman rules for the effective Lagrangian}

In this appendix we present selected Feynman rules for the six dimensional effective theory of interest to us, defined by the Lagrangian

$$
\begin{aligned}
\mathcal{L}_{\mathrm{QCD}}^{6}=\bar{\psi}(i \not D-m) \psi & -\frac{1}{2} \operatorname{tr} F_{\mu \nu} F^{\mu \nu}+\frac{i}{2} \sigma_{1} g_{s}^{3} m_{t} \bar{\psi} \gamma^{\mu} \gamma^{\nu} F_{\mu \nu} \psi \\
& +i \sigma_{2} g_{s}^{3} \bar{\psi} F_{\mu \nu} \gamma^{\mu} D^{\nu} \psi+\frac{i}{6} \gamma g_{s}^{3} \operatorname{tr}\left(F^{\mu \nu}\left[F_{\mu \lambda}, F_{\nu}{ }^{\lambda}\right]\right)
\end{aligned}
$$

We further define

$$
\begin{aligned}
F_{\mu \nu}^{a} & =\partial_{\mu} A_{\nu}^{a}-\partial_{\nu} A_{\mu}^{a}+g_{s} f^{a b c} A_{\mu}^{b} A_{\nu}^{c} \\
\sigma^{\mu \nu} & =\frac{i}{2}\left(\gamma^{\mu} \gamma^{\nu}-\gamma^{\nu} \gamma^{\mu}\right) .
\end{aligned}
$$

These rules were derived with the help of FeynCalc [50, 51] and FeynRules [52, 53]. The vertices are colour ordered and all momenta are considered to be out-going. We include the 
coupling constants here for clarity though in the main text they are stripped off.

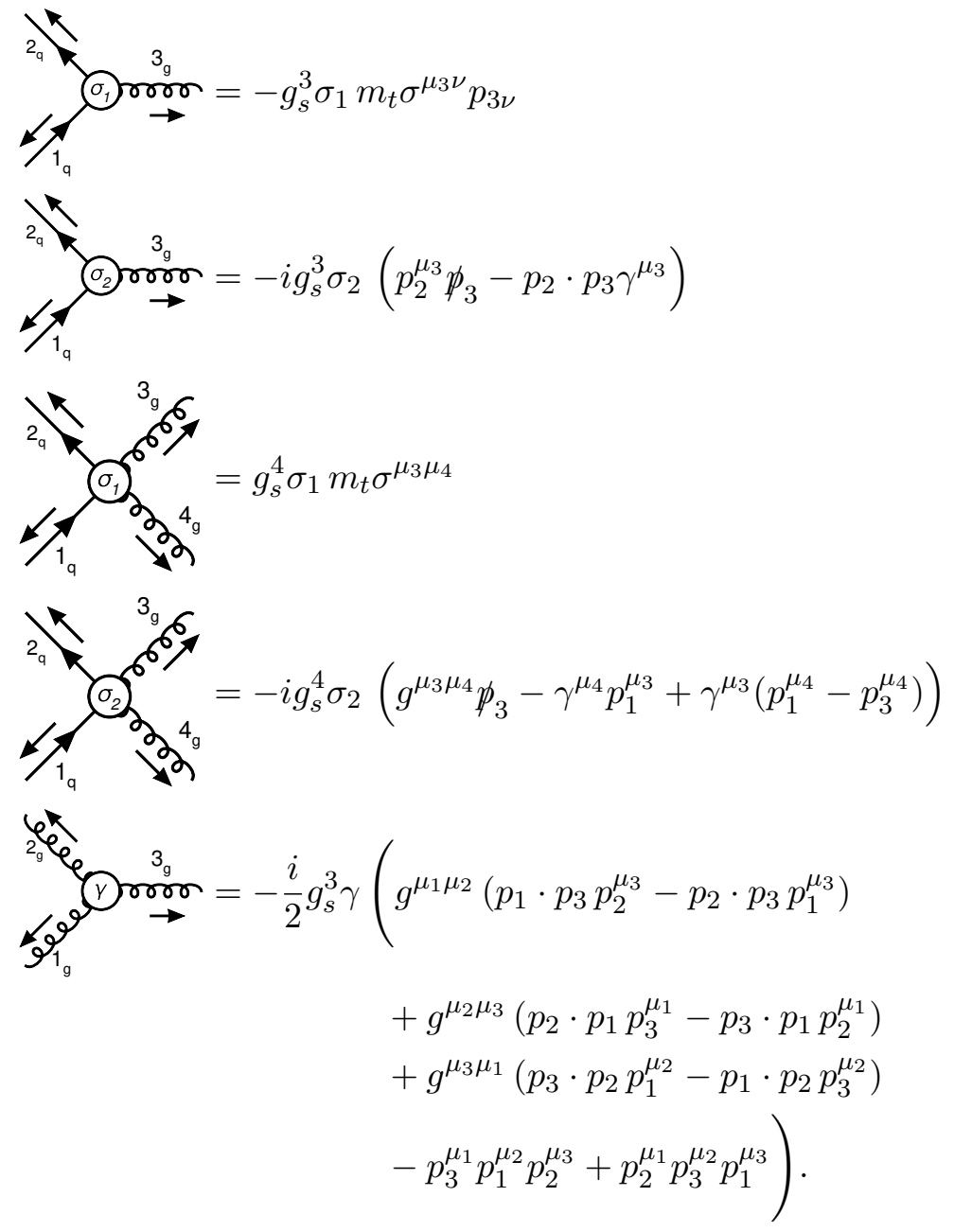

Open Access. This article is distributed under the terms of the Creative Commons Attribution License (CC-BY 4.0), which permits any use, distribution and reproduction in any medium, provided the original author(s) and source are credited.

\section{References}

[1] M. Czakon, P. Fiedler and A. Mitov, Total Top-Quark Pair-Production Cross section at Hadron Colliders Through $O\left(\alpha_{S}^{4}\right)$, Phys. Rev. Lett. 110 (2013) 252004 [arXiv:1303.6254] [INSPIRE].

[2] M. Czakon, D. Heymes and A. Mitov, High-precision differential predictions for top-quark pairs at the LHC, Phys. Rev. Lett. 116 (2016) 082003 [arXiv:1511.00549] [InSPIRE].

[3] G. Bevilacqua, H.B. Hartanto, M. Kraus and M. Worek, Top Quark Pair Production in Association with a Jet with Next-to-Leading-Order QCD Off-Shell Effects at the Large Hadron Collider, Phys. Rev. Lett. 116 (2016) 052003 [arXiv:1509. 09242] [INSPIRE].

[4] S. Höche, P. Maierhöfer, N. Moretti, S. Pozzorini and F. Siegert, Next-to-leading order QCD predictions for top-quark pair production with up to three jets, Eur. Phys. J. C 77 (2017) 145 [arXiv: 1607.06934] [INSPIRE]. 
[5] H. van Deurzen, G. Luisoni, P. Mastrolia, E. Mirabella, G. Ossola and T. Peraro, Next-to-Leading-Order QCD Corrections to Higgs Boson Production in Association with a Top Quark Pair and a Jet, Phys. Rev. Lett. 111 (2013) 171801 [arXiv:1307.8437] [INSPIRE].

[6] J.R. Andersen et al., Les Houches 2015: Physics at TeV Colliders Standard Model Working Group Report, arXiv:1605.04692 [INSPIRE].

[7] Z. Bern and A.G. Morgan, Massive loop amplitudes from unitarity, Nucl. Phys. B 467 (1996) 479 [hep-ph/9511336] [INSPIRE].

[8] R. Britto and B. Feng, Unitarity cuts with massive propagators and algebraic expressions for coefficients, Phys. Rev. D 75 (2007) 105006 [hep-ph/0612089] [INSPIRE].

[9] R. Britto, B. Feng and P. Mastrolia, Closed-Form Decomposition of One-Loop Massive Amplitudes, Phys. Rev. D 78 (2008) 025031 [arXiv: 0803.1989] [InSPIRE].

[10] J.S. Rozowsky, Feynman diagrams and cutting rules, hep-ph/9709423 [INSPIRE].

[11] S. Badger, J.M. Campbell and R.K. Ellis, QCD corrections to the hadronic production of a heavy quark pair and a W-boson including decay correlations, JHEP 03 (2011) 027 [arXiv: 1011.6647] [INSPIRE].

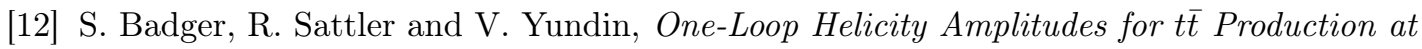
Hadron Colliders, Phys. Rev. D 83 (2011) 074020 [arXiv:1101.5947] [INSPIRE].

[13] J.M. Campbell and R.K. Ellis, Top-Quark Processes at NLO in Production and Decay, J. Phys. G 42 (2015) 015005 [arXiv: 1204.1513] [InSPIRE].

[14] Z. Bern, L.J. Dixon, D.C. Dunbar and D.A. Kosower, One loop $n$ point gauge theory amplitudes, unitarity and collinear limits, Nucl. Phys. B 425 (1994) 217 [hep-ph/9403226] [INSPIRE].

[15] Z. Bern, L.J. Dixon, D.C. Dunbar and D.A. Kosower, Fusing gauge theory tree amplitudes into loop amplitudes, Nucl. Phys. B 435 (1995) 59 [hep-ph/9409265] [InSPIRE].

[16] R. Britto, F. Cachazo and B. Feng, Generalized unitarity and one-loop amplitudes in $N=4$ super-Yang-Mills, Nucl. Phys. B 725 (2005) 275 [hep-th/0412103] [INSPIRE].

[17] R.K. Ellis, W.T. Giele, Z. Kunszt and K. Melnikov, Masses, fermions and generalized D-dimensional unitarity, Nucl. Phys. B 822 (2009) 270 [arXiv:0806.3467] [InSPIRE].

[18] R. Britto and E. Mirabella, External leg corrections in the unitarity method, JHEP 01 (2012) 045 [arXiv: 1109.5106] [INSPIRE].

[19] C. Cheung and D. O'Connell, Amplitudes and Spinor-Helicity in Six Dimensions, JHEP 07 (2009) 075 [arXiv: 0902.0981] [INSPIRE].

[20] Z. Bern, J.J. Carrasco, T. Dennen, Y.-t. Huang and H. Ita, Generalized Unitarity and Six-Dimensional Helicity, Phys. Rev. D 83 (2011) 085022 [arXiv:1010.0494] [InSPIRE].

[21] S. Davies, One-Loop QCD and Higgs to Partons Processes Using Six-Dimensional Helicity and Generalized Unitarity, Phys. Rev. D 84 (2011) 094016 [arXiv:1108. 0398] [INSPIRE].

[22] Z. Bern, L.J. Dixon and D.A. Kosower, On-Shell Methods in Perturbative QCD, Annals Phys. 322 (2007) 1587 [arXiv: 0704.2798] [INSPIRE].

[23] L.J. Dixon, A brief introduction to modern amplitude methods, arXiv:1310.5353 [INSPIRE].

[24] R.K. Ellis, Z. Kunszt, K. Melnikov and G. Zanderighi, One-loop calculations in quantum field theory: from Feynman diagrams to unitarity cuts, Phys. Rept. 518 (2012) 141 [arXiv: 1105.4319] [INSPIRE]. 
[25] G. Ossola, C.G. Papadopoulos and R. Pittau, Reducing full one-loop amplitudes to scalar integrals at the integrand level, Nucl. Phys. B 763 (2007) 147 [hep-ph/0609007] [InSPIRE].

[26] R.K. Ellis, W.T. Giele and Z. Kunszt, A Numerical Unitarity Formalism for Evaluating One-Loop Amplitudes, JHEP 03 (2008) 003 [arXiv: 0708.2398] [INSPIRE].

[27] W.T. Giele, Z. Kunszt and K. Melnikov, Full one-loop amplitudes from tree amplitudes, JHEP 04 (2008) 049 [arXiv: 0801.2237] [INSPIRE].

[28] W.L. van Neerven and J.A.M. Vermaseren, Large loop integrals, Phys. Lett. B 137 (1984) 241 [INSPIRE].

[29] R. Kleiss and W.J. Stirling, Spinor Techniques for Calculating $p \bar{p} \rightarrow W^{ \pm} / Z^{0}+$ Jets, Nucl. Phys. B 262 (1985) 235 [INSPIRE].

[30] K. Hagiwara and D. Zeppenfeld, Helicity Amplitudes for Heavy Lepton Production in $e^{+} e^{-}$ Annihilation, Nucl. Phys. B 274 (1986) 1 [InSPIRE].

[31] C. Schwinn and S. Weinzierl, Scalar diagrammatic rules for Born amplitudes in QCD, JHEP 05 (2005) 006 [hep-th/0503015] [INSPIRE].

[32] G. Rodrigo, Multigluonic scattering amplitudes of heavy quarks, JHEP 09 (2005) 079 [hep-ph/0508138] [INSPIRE].

[33] D. Maître and P. Mastrolia, S@M, a Mathematica Implementation of the Spinor-Helicity Formalism, Comput. Phys. Commun. 179 (2008) 501 [arXiv:0710.5559] [INSPIRE].

[34] R. Boels, Covariant representation theory of the Poincaré algebra and some of its extensions, JHEP 01 (2010) 010 [arXiv: 0908. 0738] [INSPIRE].

[35] T. Dennen, Y.-t. Huang and W. Siegel, Supertwistor space for 6D maximal super Yang-Mills, JHEP 04 (2010) 127 [arXiv:0910.2688] [INSPIRE].

[36] S. Caron-Huot and D. O'Connell, Spinor Helicity and Dual Conformal Symmetry in Ten Dimensions, JHEP 08 (2011) 014 [arXiv:1010.5487] [INSPIRE].

[37] T. Dennen and Y.-t. Huang, Dual Conformal Properties of Six-Dimensional Maximal Super Yang-Mills Amplitudes, JHEP 01 (2011) 140 [arXiv: 1010.5874] [INSPIRE].

[38] R.H. Boels and D. O'Connell, Simple superamplitudes in higher dimensions, JHEP 06 (2012) 163 [arXiv: 1201.2653] [INSPIRE].

[39] R.K. Ellis and G. Zanderighi, Scalar one-loop integrals for QCD, JHEP 02 (2008) 002 [arXiv:0712.1851] [INSPIRE].

[40] Z. Bern, L.J. Dixon and D.A. Kosower, One loop corrections to two quark three gluon amplitudes, Nucl. Phys. B 437 (1995) 259 [hep-ph/9409393] [inSPIRE].

[41] K. Risager Larsen, Unitarity and On-Shell Recursion Methods for Scattering Amplitudes, arXiv:0804.3310 [INSPIRE].

[42] C.F. Berger et al., An Automated Implementation of On-Shell Methods for One-Loop Amplitudes, Phys. Rev. D 78 (2008) 036003 [arXiv:0803.4180] [INSPIRE].

[43] S. Catani, S. Dittmaier and Z. Trócsányi, One loop singular behavior of QCD and SUSY QCD amplitudes with massive partons, Phys. Lett. B 500 (2001) 149 [hep-ph/0011222] [INSPIRE].

[44] M.B. Wise and E. Witten, A Diagrammatic Analysis of Some Contributions to the $\Delta I=1 / 2$ Rule, Phys. Rev. D 20 (1979) 1216 [inSPIRE]. 
[45] H.D. Politzer, Power Corrections at Short Distances, Nucl. Phys. B 172 (1980) 349 [INSPIRE].

[46] C. Arzt, Reduced effective Lagrangians, Phys. Lett. B 342 (1995) 189 [hep-ph/9304230] [INSPIRE].

[47] C. Grosse-Knetter, Effective Lagrangians with higher derivatives and equations of motion, Phys. Rev. D 49 (1994) 6709 [hep-ph/9306321] [INSPIRE].

[48] H. Simma, Equations of motion for effective Lagrangians and penguins in rare B decays, Z. Phys. C 61 (1994) 67 [hep-ph/9307274] [INSPIRE].

[49] R.N. Lee, Presenting LiteRed: a tool for the Loop InTEgrals REDuction, arXiv:1212.2685 [INSPIRE].

[50] R. Mertig, M. Böhm and A. Denner, FEYN CALC: Computer algebraic calculation of Feynman amplitudes, Comput. Phys. Commun. 64 (1991) 345 [INSPIRE].

[51] V. Shtabovenko, R. Mertig and F. Orellana, New Developments in FeynCalc 9.0, Comput. Phys. Commun. 207 (2016) 432 [arXiv:1601.01167] [inSPIRE].

[52] N.D. Christensen and C. Duhr, FeynRules - Feynman rules made easy, Comput. Phys. Commun. 180 (2009) 1614 [arXiv:0806.4194] [INSPIRE].

[53] A. Alloul, N.D. Christensen, C. Degrande, C. Duhr and B. Fuks, FeynRules 2.0 - A complete toolbox for tree-level phenomenology, Comput. Phys. Commun. 185 (2014) 2250 [arXiv: 1310.1921] [INSPIRE]. 